\title{
Comparing Density Forecasts via Weighted Likelihood Ratio Tests: Asymptotic and Bootstrap Methods
}

\author{
Raffaella Giacomini* \\ University of California, San Diego
}

June 2002

\begin{abstract}
This paper proposes and analyzes tests that can be used to compare the accuracy of alternative conditional density forecasts of a variable. The tests are also valid in the broader context of model selection based on out-of-sample predictive ability. We restrict attention to the case of density forecasts derived from non-nested parametric models, with known or estimated parameters. The evaluation makes use of scoring rules, which are loss functions defined over the density forecast and the realizations of the variable. In particular, we consider the logarithmic scoring rule, which leads to the development of asymptotic and bootstrap 'weighted likelihood ratio' tests. The name comes from the fact that the tests compare weighted averages of the scores over the available sample, as a way to focus attention on different regions of the distribution of the variable. For a uniform weight function, the asymptotic test can be interpreted as an extension of Vuong (1989)'s likelihood ratio test for non-nested hypotheses to time series data and to an out-of-sample testing framework. A Monte Carlo simulation explores the size and power properties of this last test in finite samples. An application using S\&P500 daily returns shows how the tests can be used to compare the performance of density forecasts obtained from GARCH models with different distributional assumptions.
\end{abstract}

KEYWORDS: Density forecasting, Scoring rules, Predictive Ability, Forecast comparison

J.E.L. Codes: C12, C22, C52, C53

${ }^{*}$ I am deeply indebted to Clive W. J. Granger for introducing me to this line of research and for many interesting discussions. I would also like to thank Carlos Capistran, Graham Elliott, Ivana Komunjer, Andrew Patton, Kevin Sheppard and Allan Timmermann for valuable comments. The computations in the paper were carried out in the UCSD Experimental and Computational Laboratory, for which I thank Vince Crawford. Address: Department of Economics 0508, University of California, San Diego, 9500 Gilman Dr., La Jolla, CA 92093. E-mail: rgiacomini@ucsd.edu. 


\section{Introduction}

A density forecast is an estimate of the future probability distribution of a random variable, conditional on the information available at the time the forecast is made. It thus represents a complete characterization of the uncertainty associated with the forecast, as opposed to a point forecast, which provides no information about the uncertainty of the prediction.

Density forecasting is receiving increasing attention in both macroeconomics and finance (see Tay and Wallis, 2000 for a survey). A famous example of density forecasting in macroeconomics is the 'fan-chart' of inflation and GDP published by the Bank of England and by the Sveriges Riksbank in Sweden in their quarterly Inflation Reports (for other examples of density forecasting in macroeconomics, see also Diebold, Tay and Wallis, 1999 and Clements and Smith, 2000). In finance, where the wide availability of data and the increasing computational power make it possible to produce more accurate estimates of densities, the examples are numerous. Leading cases are in risk management, where forecasts of portfolio distributions are issued with the purpose of tracking measures of portfolio risk such as the Value-at-Risk (see, e.g., Duffie and Pan, 1996) or the Expected Shorfall (see, e.g., Artzner et al., 1997). Another example is the extraction of density forecasts from option price data (see, e.g. Soderlind and Svensson, 1997). The vast literature on forecasting volatility with GARCH-type models (see Bollerslev, Engle and Nelson, 1994) and its extensions to forecasting higher moments of the conditional distribution (see Hansen, 1994) can also be seen as precursors to density forecasting. The use of sophisticated distributions for the standardized residuals of a GARCH model and the modeling of time dependence in higher moments is in many cases an attempt to capture relevant features of the data to better approximate the true distribution of the variable. Finally, a focus on densities is the central issue in the literature on copula modeling and forecasting, that is gaining interest in financial econometrics (see Patton, 2001).

With density forecasting becoming more and more widespread in applied econometrics, it is necessary to develop reliable techniques to evaluate the forecasts' performance. A popular method for evaluating a sequence of conditional density forecasts was proposed by Diebold, Gunther and Tay (1998). These authors suggested evaluating a sequence of density forecasts by considering the probability integral transforms $\left(z_{t}\right)$ of the realizations of the variable with respect to the forecast densities. If the density forecasts coincide with the true conditional densities, the sequence $\left\{z_{t}\right\}$ is independent and identically distributed (i.i.d.) $U(0,1)$. While Diebold et al. (1998) adopted mainly qualitative tools for testing the i.i.d. $U(0,1)$ behavior of the transformed data, formal tests of the same hypothesis have been recently suggested by Berkowitz (2000), Hong (2000) and Hong and White 
(2000).

Even with rigorous testing procedures of the i.i.d. $U(0,1)$ hypothesis available, it is important to emphasize that the Diebold et al. (1998)'s method is only valid in absolute terms, that is, to evaluate the 'goodness' of a particular sequence of density forecasts, relative to the data-generating process. In practice, it is likely that any econometric model used to produce the sequence of density forecasts is misspecified. In this situation a more relevant question is how to decide which one of two (or more) given alternative density forecasts is preferable. The issue of comparative evaluation of density forecasts has not yet been explored from a methodological point of view, but, in spite of this, there are a few examples of empirical research that attempt to compare density forecasts. The Diebold et al. (1998) technique, in particular, has inspired a number of applications where alternative density forecasts are evaluated by constructing their corresponding sequences of probability integral transforms and comparing their relative distance to the uniform distribution, see, e.g., Clements and Smith (2000) and Weigend and Shi (2000). This distance is in most situations assessed only through visual inspection, and no formal testing is utilized. This paper attempts to fill the gap in the literature and propose formal tests that can be utilized to rank alternative density forecasts.

In the paper, we restrict attention to an environment in which two possibly misspecified, parametric models are used to generate conditional density forecasts for the variable of interest. The models are assumed to be non-nested and the parameters of the densities are either known or estimated. We emphasize that even though the paper focuses on a density forecasting environment, the techniques proposed can be used in the more general context of model selection. In this case, two competing models will be analyzed in terms of their ex-post predictive performance, in an outof-sample evaluation exercise that compares forecasts of the entire conditional density, rather than simple point forecasts implied by the two models. As such, our tests can be used in conjunction with Diebold-Mariano (1995) type of tests, that compare models according to their (point) forecasting accuracy or in terms of the relative loss implied by some economically meaningful criterion. Even though our focus in the paper is on univariate densities, all the tests can be easily generalized to the multivariate case, which is for example relevant in the context of evaluation and selection of copula models.

We measure the relative accuracy of density forecasts by so-called 'scoring rules', which are loss functions defined over the density forecast and the outcome of the variable. In particular, we restrict attention to the logarithmic scoring rule, and suggest ranking the forecasts according to the relative magnitude of a weighted average of the scores over the available sample. We show that the use 
of a weighted average allows the user to compare the performance of density forecasts in different regions of the unconditional distribution of the variable, distinguishing for example predictive ability in 'normal' days from that in 'extreme' days. The use of a simple average yields instead the standard likelihood ratio test for comparison of non-nested models proposed by Vuong (1989), extended to the case of time series data and to an out-of-sample testing framework.

The paper is organized as follows. Section 2 introduces the notation and the assumptions utilized in the paper. In Section 3, we briefly discuss loss functions for the evaluation of density forecasts. The class of loss functions proposed leads to the development of asymptotic and bootstrap weighted likelihood ratio tests, discussed in Section 4. Section 5 derives an extension of the standard likelihood ratio test for non-nested hypotheses proposed by Vuong (1989) to time series data and to an out-ofsample framework. A bootstrap and a bootstrap- $t$ likelihood ratio tests are also considered, and the small sample properties of the tests are analyzed in a Monte Carlo simulation in Section 6. All the tests proposed in the paper are used in Section 7 to compare density forecasts for the S\&P 500 daily returns obtained from GARCH models with different distributional assumptions. Finally, Section 8 concludes.

\section{Description of environment}

\subsection{Notation}

For simplicity we will restrict attention to the univariate case. The extension to multivariate is relatively straightforward. The density forecasts are based on two alternative conditional models $F_{\theta} \equiv\left\{f\left(y_{t+1} \mid \Omega_{t} ; \theta\right) ; \theta \in \Theta\right\}$ and $G_{\gamma} \equiv\left\{g\left(y_{t+1} \mid \Omega_{t} ; \gamma\right) ; \gamma \in \Gamma\right\}$. The parameter spaces $\Theta$ and $\Gamma$ are respectively $k_{1}$ and $k_{2}$ dimensional. The forecasts are conditional on the information set $\Omega_{t}=$ $\left\{y_{t-j}, x_{t+1-j} ; j \geq 0\right\}$, containing the past history of the variable of interest $Y_{t}$ and possibly the history of other explanatory variables denoted jointly as $X_{t+1}$. We point out that the two models are not restricted to use the same selection of explanatory variables in their specifications. We consider a recursive forecasting scheme. The available sample of size $T$ is divided in two parts, with the first $R$ data used for estimation and the last $n$ for out-of-sample evaluation. The first forecasts are formed using data from 1 to $R$, the second using data 1 to $R+1$ and so forth. The last forecasts are produced by estimating the models on data from 1 to $R+n-1 \equiv T-1$. Let $\hat{\theta}_{t}$ and $\hat{\gamma}_{t}$ denote the estimators based on data from 1 to $t$. This procedure will generate two sequences of $n$ density forecasts

$$
\left\{f\left(y_{t+1} \mid \Omega_{t} ; \hat{\theta}_{t}\right)\right\}_{t=R}^{T-1} \text { and }\left\{g\left(y_{t+1} \mid \Omega_{t} ; \hat{\gamma}_{t}\right)\right\}_{t=R}^{T-1}
$$


for the variables $Y_{R+1}, \ldots, Y_{t}$. We let $\theta^{*}$ and $\gamma^{*}$ denote the probability limits respectively of $\hat{\theta}_{t}$ and $\hat{\gamma}_{t}$.

\subsection{Assumptions}

The first set of assumptions, collected in Assumption 1, is related to the regularity conditions utilized by West (1996). The assumptions only stated in terms of $f$ and $\theta$ implicitly hold for $g$ and $\gamma$. The symbol $\nabla_{\theta}^{k}$ will denote the $k$-th derivative operator with respect to $\theta$. The function $w(\cdot)$ appearing in Assumption 1-(f) is a weight function used in the development of weighted likelihood ratio tests, whose meaning and use will be made more precise in Section 3.

Assumption 1. Let $N$ be an open neighborhood of $\theta^{*}:$ (a) $f\left(y_{t+1} \mid \Omega_{t}, \cdot\right)$ is continuously differentiable of order 2 on $N$.

(b) There exists a constant $D<\infty$ such that for all $t, \sup _{\theta \in N}\left|\nabla_{\theta}^{2} \log f\left(Y_{t+1} \mid \Omega_{t}, \theta\right)\right|<m_{t}$ for a measurable $m_{t}$ that satisfies $E m_{t}<D$.

(c) The estimate $\hat{\theta}_{t}$ satisfies $\hat{\theta}_{t}-\theta^{*}=B^{f}(t) A^{f}(t)$, where $B^{f}(t)$ is $k_{1} \times q$ and $A^{f}(t)$ is $q \times 1$, with $B^{f}(t) \stackrel{a . s .}{\rightarrow} B^{f}, B^{f}$ matrix of rank $k_{1}$ and $A^{f}(t)=t^{-1} \sum_{s=1}^{t} a_{s}^{f}\left(\theta^{*}\right)$ for a $q \times 1$ orthogonality condition $a_{s}^{f}\left(\theta^{*}\right)$ such that $E a_{s}^{f}\left(\theta^{*}\right)=0$.

(d) For some $d, d^{\prime}, d^{\prime \prime}>1, \sup _{t} E\left|\log f\left(Y_{t+1} \mid \Omega_{t}, \theta^{*}\right)\right|^{4 d}, \sup _{t} E\left|\nabla_{\theta i} \log f\left(Y_{t+1} \mid \Omega_{t}, \theta^{*}\right)\right|^{4 d^{\prime}}, \sup _{t} E\left|a_{t}^{f \prime}\right|^{4 d^{\prime \prime}}$ $<\infty$, for all $i$, where $\nabla_{\theta i}$ is the $i$-th component of the gradient.

(e) $\left\{Y_{t}\right\}$ is strong mixing, with mixing coefficients of size $-3 d /(d-1)$.

(f) $\left[w\left(Y_{t+1}\right) \log f\left(Y_{t+1} \mid \Omega_{t}, \theta^{*}\right),\left(w\left(Y_{t+1}\right) \nabla_{\theta} \log f\left(Y_{t+1} \mid \Omega_{t}, \theta^{*}\right)\right)^{\prime}, a_{t}^{f \prime}\right]^{\prime}$ is covariance stationary.

Assumption 1 imposes conditions on the density models, the weight function, the estimation procedure and the data-generating process of the random variable $Y_{t}$. The restrictions are fairly standard, and allow for application of the results to a wide range of situations that arise in practice. In particular, we demand the use of smooth density functions for the forecast models, but this requirement could be relaxed along the lines of McCracken (2000). We also require existence of at least four moments of the log-likelihoods and the scores. This requirement, in general, depends on both the density models and the true density, and its plausibility should thus be verified on a case by case basis. The parameters of the models can be estimated by a variety of linear and nonlinear techniques, including Maximum Likelihood, OLS and GMM. The restrictions on memory and heterogeneity of the data-generating process still allow for conditional heterogeneity and serial dependence. $^{1}$

\footnotetext{
${ }^{1}$ The assumption of covariance stationarity (Assumption 1-f) is mainly imposed for convenience in estimating the asymptotic variance matrix. This assumption could be relaxed, at the price of increased complexity (see Rivers and
} 
Assumption 2. (a) The conditional models $F_{\theta}$ and $G_{\gamma}$ are non-nested: $F_{\theta} \nsubseteq G_{\gamma}$ and $G_{\gamma} \nsubseteq F_{\theta}$. (b) $f\left(\cdot \mid \cdot ; \theta^{*}\right) \neq g\left(\cdot \mid \cdot ; \gamma^{*}\right)$.

Part (a) indicates that the models can be either strictly non-nested $\left(F_{\theta} \cap G_{\gamma}=\emptyset\right)$ or overlapping $\left(F_{\theta} \cap G_{\gamma} \neq \emptyset\right.$ but $F_{\theta} \nsubseteq G_{\gamma}$ and $\left.G_{\gamma} \nsubseteq F_{\theta}\right)$. Examples of strictly non-nested densities are, e.g., the normal and the lognormal, the Student's $t$ and the Generalized Error Distribution $(G E D)$ with finite degrees of freedom parameters. Alternatively, non-nestedness can be achieved when both $f$ and $g$ belong to the same family of distributions, but the models specify non-nested expressions for, say, the conditional mean or variance. An example is the case of two different non-linear specifications that cannot be obtained from each other, or of models that utilize different explanatory variables. Overlapping arises when the two models are not nested but still possess some common elements, as in the case of conditional moment equations that depend on some common explanatory variable or of two families of distributions that both nest the normal. For a more complete discussion, see Vuong (1989).

Part (b) is relevant in the case of overlapping models. It requires the density forecasts to be distinct only when evaluated at the respective probability limits of the parameters. In practice, unless the probability limits of the parameter estimates are known a priori, one will have to pre-test for condition (b). In the example where $f$ and $g$ belong to the same family of distributions and specify conditional moment equations that depend on common and non-common variables, one should verify that at least one of the coefficients on the non-common variables is significantly different from zero ${ }^{2}$. In this case, it is guaranteed that the two density forecasts evaluated at the probability limits of the parameters are distinct.

Assumption 3. As $T \rightarrow \infty, R, n \rightarrow \infty$ and $\lim _{T \rightarrow \infty}(n / R)=\pi, 0 \leq \pi<\infty$.

Assumption 3 allows the in-sample and the out-of-sample sizes to diverge at the same rate, or the in-sample size to grow faster than the out-of-sample. This assumption concerns the way the asymptotic distribution is achieved. In particular, letting the in-sample and the out-of-sample diverge at the same rate is a way to state that the asymptotic distribution of the test statistics will take into account the uncertainty due to estimated parameters. Imposing $\pi=0$, on the other hand, is an artificial way to ensure that estimation uncertainty will not affect the asymptotic distribution.

\footnotetext{
Vuong, 1999).

${ }^{2}$ In this case, the following test will not have exact size $\alpha$. Instead, $\alpha$ will represent an upper bound on the actual size of the test.
} 


\section{Loss functions and density forecasting}

There is a large literature on loss functions for evaluation of point forecasts (e.g., Christoffersen and Diebold, 1997). In this section, we explore the possibility of incorporating loss functions into the evaluation of density forecasts, and argue that the standard framework of loss functions for forecast evaluation is not appropriate when the object to be forecasted is a conditional density.

The incorporation of loss functions into the forecasting problem has until now focused on the definition of classes of loss functions of the form $L\left(\hat{y}_{t, \tau}, y_{t+\tau}\right)$, where $\hat{y}_{t, \tau}$ is a $\tau$-step-ahead point forecast of $Y_{t+\tau}$ and $y_{t+\tau}$ is the realization of the variable. In the vast majority of cases, the loss function is assumed to only depend on the forecast error, as for quadratic loss or general asymmetric loss (e.g., Christoffersen and Diebold, 1997, Weiss, 1996). Weiss (1996) shows that, in this framework, the optimal predictor is some summary measure of the true conditional density of the variable $Y_{t+\tau}$ (the mean for quadratic loss, the median for absolute error loss, etc.). This means that a user with, say, a quadratic loss function will only care about the accuracy of the mean prediction and will be indifferent among density forecasts that yield the same forecast for the conditional mean. As a consequence, in this situation it becomes unnecessary to issue a density forecast in the first place, and the forecaster should only concentrate on accurately forecasting the relevant summary measure of the true density. The discussion of loss functions relevant for density forecasting must thus involve a shift of focus.

Since a density forecast can be seen as a collection of probabilities assigned by the forecaster to all attainable events, the tools developed in the probability forecasting evaluation literature can be readily employed. In particular, we will make use of so-called 'scoring rules' for evaluation of density forecasts. Scoring rules (see, inter alia, Winkler, 1967, Blattenberger and Lad, 1988, Diebold and Lopez, 1996, Lopez, 2001) are loss functions whose arguments are the density forecast and the actual outcome of the variable, and they play an important role in probability forecasting both in an ex-ante and ex-post sense. Ex-ante, scoring rules are used to elicit subjective probabilities from experts. Ex-post, they provide a tool for evaluation and comparison of alternative probability forecasts. Common choices of scoring rules for continuous distributions (e.g., Matheson and Winkler, 1976) are the quadratic (or Brier score), logarithmic and spherical scores, respectively given by

$$
\begin{aligned}
& S(f, y)=2 f(y)-\int_{-\infty}^{+\infty}(f(u))^{2} d u \\
& S(f, y)=\log f(y) \\
& S(f, y)=f(y) /\left(\int_{-\infty}^{+\infty}(f(u))^{2} d u\right)^{1 / 2}
\end{aligned}
$$


where $y$ is the observed value of the variable and $f(\cdot)$ the density forecast. ${ }^{3}$

In this paper, we adopt an ex-post perspective, and restrict attention to the logarithmic scoring rule (3) as a tool for comparing alternative density forecasts. Intuitively, the logarithmic score rewards a density forecast which assigns high probability to the event that actually occurred. The logarithmic score is also mathematically convenient, being the only scoring rule that is solely a function of the value of the density at the realization of the variable.

When a sequence of alternative density forecasts and of observed values of the variable is available, one can rank the density forecasts by comparing the average realized scores for each forecast. For the two sequences of density forecasts $f$ and $g$ introduced in Section 2.1, one would compute the average scores over the out-of-sample period as $\bar{S}_{f}=n^{-1} \sum_{t=R}^{T-1} \log f\left(y_{t+1} \mid \Omega_{t} ; \hat{\theta}_{t}\right)$ and $\bar{S}_{g}=$ $n^{-1} \sum_{t=R}^{T-1} \log g\left(y_{t+1} \mid \Omega_{t} ; \hat{\gamma}_{t}\right)$, and select the forecast yielding the highest score.

In this paper, we suggest a more general approach which involves considering a weighted average of the scores over the out-of-sample period. The idea is that a user might be especially interested in a density forecast that is accurate in predicting events that lay in a particular region of the unconditional distribution of the variable of interest. An example could be a user who only cares about predicting (loosely defined) tail events, as in the case when different investment strategies or policy implications would arise if the future realizations of the variable fall into the tails of the distribution. If the user is presented with two alternative density forecasts, he might then want to place greater emphasis on the performance of the competing models in the tails of the distribution, and less emphasis on what happens in the center. Another situation that might be of interest is a focus on predicting events that fall near the unconditional mean of the variable, as a way to ignore the influence of possible outliers on predictive performance. Finally, one might want to separate the predictive performance of the models in the right and in the left tail of the distribution, as in the case, e.g., of forecasting models for risk management, where losses have different implications than gains.

For each of the above situations, we can define an appropriate weight function $w(\cdot)$ and compare the weighted average scores $\bar{S}_{f}^{w}=n^{-1} \sum_{t=R}^{T-1} w\left(y_{t+1}\right) \log f\left(y_{t+1} \mid \Omega_{t} ; \hat{\theta}_{t}\right)$ and $\bar{S}_{g}^{w}=n^{-1} \sum_{t=R}^{T-1} w\left(y_{t+1}\right)$ $\log g\left(y_{t+1} \mid \Omega_{t} ; \hat{\gamma}_{t}\right)$. The weight function $w(\cdot)$ can be arbitrarily chosen by the researcher to select the desired region of the unconditional distribution of $Y_{t}$. The only requirements imposed on the weight function are that $w: \mathbb{R} \rightarrow(0,1]$ and that it be twice continuously differentiable on $\mathbb{R}$. For example,

\footnotetext{
${ }^{3} \mathrm{~A}$ scoring rule is usually expressed as a gain, rather than a loss. In spite of this, we will continue referring to scoring rules as loss functions.
} 
when the data have unconditional mean 0 and variance 1 , one could consider the following weight functions.

- Center of distribution: $w_{1}(y)=\phi(y), \phi$ standard normal density function (or pdf)

- Tails of distribution: $w_{2}(y)=1-\phi(y) / \phi(0), \phi$ standard normal pdf

- Right tail: $w_{3}(y)=\Phi(y), \Phi$ standard normal distribution function (or cdf)

- Left tail: $w_{4}(y)=1-\Phi(y), \Phi$ standard normal cdf

Plots of $w_{1}-w_{4}$ are shown in Figure 1.

[FIGURE 1 HERE]

A formal test for comparing the weighted average logarithmic scores is proposed in the following section.

\section{Weighted likelihood ratio tests}

For a given weight function $w(\cdot)$ and two alternative conditional density forecasts $f(\cdot \mid \cdot)$ and $g(\cdot \mid \cdot)$ for $Y_{t+1}$, consider

$$
\left.W L R^{*} \equiv E\left[w\left(Y_{t+1}\right)\left(\log f\left(Y_{t+1} \mid \Omega_{t} ; \theta^{*}\right)\right)-\log g\left(Y_{t+1} \mid \Omega_{t} ; \gamma^{*}\right)\right)\right]
$$

A test for equal weighted average logarithmic scores of density forecasts $f$ and $g$ can be formulated as

$$
\begin{aligned}
H_{0}: & W L R^{*}=0 \text { against } \\
H_{f} & : \quad W L R^{*}>0 \text { or } \\
H_{g} & : \quad W L R^{*}<0,
\end{aligned}
$$

where the two alternative hypotheses respectively indicate that $f$ is better than $g$ or that $g$ is better than $f$. We call a test of $H_{0}$ a 'weighted likelihood ratio' test.

The test above allows one to rank the two density forecasts for a particular choice of weight function. To reduce dependence on the functional form chosen for the weight function, one might consider generalizing the test (6) to take into account possibly different specifications for $w(\cdot)$. For example, if the null hypothesis is rejected in favour of one of the alternatives, say $H_{f}$, a test of superior 
predictive ability of density forecast $f$ relative to $g$ could be constructed by considering a sequence of $J$ weight functions $\left\{w_{j}(\cdot)\right\}_{j=1}^{J}$ spanning the whole support of the unconditional distribution of $Y_{t}$ and testing whether $W L R^{*}>0$ for all $w_{j}$. The theoretical underpinnings of such a test are not further considered in this paper, and are left for future research.

The expression for $W L R^{*}$ in (5) depends on the unknown expectation $E[\cdot]$ and probability limits $\theta^{*}$ and $\gamma^{*}$. We consider estimating $W L R^{*}$ by the out-of-sample analogue

$$
W L R_{n}=n^{-1} \sum_{t=R}^{T-1} w d_{t+1}\left(\hat{\beta}_{t}\right),
$$

where $w d_{t+1}\left(\hat{\beta}_{t}\right) \equiv w\left(y_{t+1}\right)\left[\log f\left(y_{t+1} \mid \Omega_{t} ; \hat{\theta}_{t}\right)-\log g\left(y_{t+1} \mid \Omega_{t} ; \hat{\gamma}_{t}\right)\right], \hat{\beta}_{t} \equiv\left(\hat{\theta}_{t}^{\prime}, \hat{\gamma}_{t}^{\prime}\right)^{\prime}$ and $\left\{y_{t}\right\}_{t=R+1}^{T}$ are the realizations of the variable over the out-of-sample period.

\subsection{Asymptotic weighted likelihood ratio test}

An asymptotic test of hypothesis $H_{0}$ can be derived using the framework developed by West (1996). The test will rely on asymptotic normality of the test statistic and the asymptotic variance will incorporate terms that reflect parameter estimation uncertainty. Before proceeding, it might be useful to recall Assumption 1-c), which introduces some notation regarding the parameter estimates and Assumption 3, which defines the constant $\pi$ that will appear in the asymptotic variance of the test statistic. We will also make use of the following notation.

$$
\begin{aligned}
& q_{t+1}(\beta) \equiv\left(\begin{array}{c}
w\left(Y_{t+1}\right) \log f\left(Y_{t+1} \mid \Omega_{t} ; \theta\right) \\
w\left(Y_{t+1}\right) \log g\left(Y_{t+1} \mid \Omega_{t} ; \gamma\right)
\end{array}\right), a_{t}(\beta) \equiv\left(\begin{array}{c}
a_{t}^{f}(\theta) \\
a_{t}^{g}(\gamma)
\end{array}\right) \\
& \delta_{q q}(j)=E\left[\left(q_{t}\left(\beta^{*}\right)-E q_{t}\left(\beta^{*}\right)\right)\left(q_{t-j}\left(\beta^{*}\right)-E q_{t}\left(\beta^{*}\right)\right)^{\prime}\right] \\
& \delta_{q a}(j)=E\left[\left(q_{t}\left(\beta^{*}\right)-E q_{t}\left(\beta^{*}\right)\right) a_{t-j}\left(\beta^{*}\right)^{\prime}\right] \\
& \delta_{a a}(j)=E\left[a_{t}\left(\beta^{*}\right) a_{t-j}\left(\beta^{*}\right)^{\prime}\right] \\
& S_{q q}=\sum_{j=-\infty}^{\infty} \delta_{q q}(j), \quad S_{q a}=\sum_{j=-\infty}^{\infty} \delta_{q a}(j), \quad S_{a a}=\sum_{j=-\infty}^{\infty} \delta_{a a}(j) \\
& F \equiv E\left(\begin{array}{cc}
w\left(Y_{t+1}\right) \nabla_{\theta} \log f\left(Y_{t+1} \mid \Omega_{t} ; \theta^{*}\right) & 0 \\
0 & w\left(Y_{t+1}\right) \nabla_{\gamma} \log g\left(Y_{t+1} \mid \Omega_{t} ; \gamma^{*}\right)
\end{array}\right), B \equiv\left(\begin{array}{cc}
B^{f} & 0 \\
0 & B^{g}
\end{array}\right) \\
& \Pi \equiv 1-\pi^{-1} \ln (1+\pi) \text { for } 0<\pi<\infty, \Pi \equiv 0 \text { for } \pi=0 \\
& \Sigma \equiv S_{q q}+\Pi\left(F B S_{q a}^{\prime}+S_{q a} B^{\prime} F^{\prime}\right)+2 \Pi F B S_{a a} B^{\prime} F^{\prime} .
\end{aligned}
$$

The following result provides the asymptotic weighted likelihood ratio test. 
Theorem 1 (Weighted Likelihood Ratio Test) Given Assumptions 1, 2, 3, $\sqrt{n}\left(W L R_{n}-W L R^{*}\right) \stackrel{D}{\rightarrow}$ $N\left(0, \sigma^{2}\right)$, where $\sigma^{2}$ is given by

$$
\sigma^{2}=\iota \Sigma \iota^{\prime}, \text { with } \iota=(1,-1)
$$

and $\Sigma$ is defined in (8). Let $\hat{\sigma}_{n}^{2}$ be a consistent estimator ${ }^{4}$ of $\sigma^{2}$, then

(i) under $H_{0}: \sqrt{n} W L R_{n} / \hat{\sigma}_{n} \stackrel{D}{\rightarrow} N(0,1)$

(ii) under $H_{f}: \sqrt{n} W L R_{n} / \hat{\sigma}_{n} \stackrel{a . s .}{\rightarrow}+\infty$

(iii) under $H_{g}: \sqrt{n} W L R_{n} / \hat{\sigma}_{n} \stackrel{a . s .}{\rightarrow}-\infty$.

For a desired level of confidence, one would first choose the corresponding critical value $c$ from the standard normal distribution. If $\left|\sqrt{n} W L R_{n} / \hat{\sigma}_{n}\right| \leq c$ one would conclude that the weighted average scores for the two density forecasts are not significantly different. If instead $\left|\sqrt{n} W L R_{n} / \hat{\sigma}_{n}\right| \geq c$, the null would be rejected in favour of $H_{f}$ (if $W L R_{n}$ is positive) or $H_{g}$ (if $W L R_{n}$ is negative). The test proposed has correct asymptotic size and is consistent, as reflected by the fact that the test statistic has a distribution that does not depend on the parameters under the null hypothesis, and it diverges under the alternative.

\subsection{Bootstrap weighted likelihood ratio test}

While the computation of the asymptotic test in the previous section can be quite involved, a test that is easier to implement can be derived utilizing the bootstrap. The test is derived by resampling the test statistic $W L R_{n}$ (1), in the following way.

A bootstrap artificial sample of size $n$ is obtained by selecting random indexes $\tau(t), t=R, \ldots, T-1$ and considering the relative sequence of out-of-sample weighted likelihood ratios $\left\{w d_{\tau(t)+1}\left(\hat{\beta}_{\tau(t)}\right) ; t=\right.$ $R, \ldots, T-1\}$. One can create $B$ such artificial samples and for each calculate the resampled test statistic as

$$
W L R_{n}^{b} \equiv n^{-1} \sum_{t=R}^{T-1} w d_{\tau(t)+1}\left(\hat{\beta}_{\tau(t)}\right), \quad b=1, \ldots, B .
$$

There are now several available techniques to do resampling when the data are dependent, as in the time-series case. Popular examples are the moving blocks bootstrap of Künsch (1989) and Liu and Singh (1992) and the stationary bootstrap of Politis and Romano (1994). In the following, we focus

\footnotetext{
${ }^{4} \mathrm{~A}$ consistent estimate of the asymptotic variance can be obtained using kernel-based estimators of each component of $\Sigma$, such as the Newey-West (1987) estimators. $\Pi$ can be estimated by $1-(R / n) \ln (1+n / R)$. See West (1996) or McCracken (2000) for a more thorough discussion on how to estimate each component of the asymptotic variance.
} 
attention on the Politis and Romano (1994) stationary bootstrap, but in principle other techniques can be used. The idea behind the stationary bootstrap is to resample blocks of random length, where the length of each block has a geometric distribution. Under some conditions on the growth rate of the average block length, Politis and Romano (1994) show that the stationary bootstrap resampling scheme satisfies desirable consistency and weak convergence properties. See also White (2000, p. 1104) for a description of how to implement the stationary bootstrap.

A bootstrap confidence interval for the weighted likelihood ratio statistic $W L R_{n}$ can be obtained in many different ways (see Shao and Tu, 1995 for a discussion). We consider for simplicity an equal-tailed $(1-\alpha) 100 \%$ confidence interval for $W L R_{n}$ obtained as

$$
C I=\left[W L R_{n}-q^{*}(1-\alpha / 2), W L R_{n}-q^{*}(\alpha / 2)\right]
$$

where $q^{*}(\alpha / 2)$ and $q^{*}(1-\alpha / 2)$ are respectively the $\alpha / 2$ and $1-\alpha / 2$ quantiles of the empirical distribution of $W L R_{n}^{b}-W L R_{n}, b=1, \ldots, B$. If $0 \notin C I$ we can reject the null hypothesis (11) of equal weighted average score of density forecasts $f$ and $g$, in favour of $f$ (if $W L R_{n}>0$ ) or in favour of $g$ (if $\left.W L R_{n}<0\right)$, at a confidence level $\alpha$. The validity of this procedure rests on the assumption that the distribution of $\sqrt{n}\left(W L R_{n}^{b}-W L R_{n}\right)$, conditional on $\left\{Y_{R+1}, \ldots, Y_{T}\right\}$ converges to the distribution of $\sqrt{n}\left(W L R_{n}-W L R^{*}\right)$, as $n$ increases. This claim is proven by Politis and Romano (1994)'s Theorem 2 for the case when the resampled statistic depends on known parameters. In the presence of $\hat{\beta}_{\tau(t)}$ in (9), the validity of the bootstrap is obtained at the cost of imposing stronger conditions on the convergence of the parameter estimators to their probability limits and on the relative growth rates of the in-sample and the out-of-sample sizes. This point is argued by White (2000), to whom the reader is referred for a rigorous treatment. For our purposes, it suffices to add the following assumptions to the ones presented in Section 2.

Assumption 4: $\hat{\beta}_{T}$ obeys a law of the iterated logarithm.

Assumption 5: $(n / R) \log \log R \rightarrow 0$ as $T \rightarrow \infty$.

Notice that Assumption 5 is effectively a strengthening of Assumption 3. To guarantee validity of the bootstrap approximation one must thus impose a condition on the relative rate of divergence of $R$ and $n$. Under Assumptions 1, 2, 4 and 5, Theorem 2.3 of White (2000) guarantees validity of the bootstrap weighted likelihood ratio test. 


\section{Likelihood ratio tests}

In this section we show how a standard likelihood ratio test can be derived as a special case of the tests analyzed in the previous section, when the weight function is identically one. We make a point of treating this case separately because the computations become particularly simple when the parameters of the density forecast are estimated by maximum likelihood. Together with the asymptotic and bootstrap tests already proposed for the general weight function case, we further consider a bootstrap- $t$ test.

The asymptotic test proposed in this section is related to Vuong (1989)'s likelihood ratio test for non-nested hypotheses. In that case, the comparison of alternative models is performed in-sample and under the assumption of independence and identical distribution of the variable of interest. In contrast, our approach focuses on the out-of-sample evaluation of density models, and we allow the variable to be characterized by conditional heterogeneity and serial dependence.

\subsection{Asymptotic likelihood ratio test}

When the weight function is $w(y)=1$ a test for equal average likelihood scores of density forecasts $f$ and $g$ can be formulated as

$$
\begin{aligned}
& H_{0}: L R^{*}=0 \text { against } \\
& H_{f}: L R^{*}>0 \text { or } \\
& H_{g}: L R^{*}<0
\end{aligned}
$$

where $L R^{*}=E\left[\log f\left(Y_{t+1} \mid \Omega_{t} ; \theta^{*}\right)-\log g\left(Y_{t+1} \mid \Omega_{t} ; \gamma^{*}\right)\right]$. Similarly to the developments in Section 4 , we estimate $L R^{*}$ by the out-of-sample mean

$$
L R_{n}=n^{-1} \sum_{t=R}^{T-1}\left[\log f\left(y_{t+1} \mid \Omega_{t} ; \hat{\theta}_{t}\right)-\log g\left(y_{t+1} \mid \Omega_{t} ; \hat{\gamma}_{t}\right)\right]
$$

where $\left\{y_{t}\right\}_{t=R+1}^{T}$ are the realizations of the variable while $\hat{\theta}_{t}$ and $\hat{\gamma}_{t}$ are Maximum Likelihood Estimators (MLE). In this section, we further assume that forecasts $f$ and $g$ make use of the same set of explanatory variables.

Let $d_{t+1}\left(\beta^{*}\right) \equiv \log f\left(Y_{t+1} \mid \Omega_{t} ; \theta^{*}\right)-\log g\left(Y_{t+1} \mid \Omega_{t} ; \gamma^{*}\right), \beta^{*}=\left(\theta^{* \prime}, \gamma^{* \prime}\right)^{\prime}$ and define

$$
\begin{aligned}
\sigma^{2} & \equiv \sum_{j=-\infty}^{+\infty} \delta_{d d}(j), \text { where } \\
\delta_{d d}(j) & =E\left[\left(d_{t}\left(\beta^{*}\right)-E d_{t}\left(\beta^{*}\right)\right)\left(d_{t-j}\left(\beta^{*}\right)-E d_{t}\left(\beta^{*}\right)\right)^{\prime}\right]
\end{aligned}
$$


The following result provides the asymptotic likelihood ratio test.

Theorem 2 (Likelihood Ratio Test) Given Assumptions 1, 2, 3, $\sqrt{n}\left(L R_{n}-L R^{*}\right) \stackrel{D}{\rightarrow} N\left(0, \sigma^{2}\right)$. Let $\hat{\sigma}_{n}^{2}$ be a consistent estimator of $\sigma^{2}$, then

(i) under $H_{0}: \sqrt{n} L R_{n} / \hat{\sigma}_{n} \stackrel{D}{\rightarrow} N(0,1)$

(ii) under $H_{f}: \sqrt{n} L R_{n} / \hat{\sigma}_{n} \stackrel{\text { a.s. }}{\rightarrow}+\infty$

(iii) under $H_{g}: \sqrt{n} L R_{n} / \hat{\sigma}_{n} \stackrel{\text { a.s. }}{\longrightarrow}-\infty$.

The expression for the asymptotic variance (13) reveals that estimation uncertainty is asymptotically irrelevant under the assumptions of the theorem. The asymptotic variance is in fact the same that would have been obtained had the parameters been known (as assumed by Diebold and Mariano, 1995), and it coincides (apart from a scale factor) with the spectral density of the variable $\left\{d_{t}\left(\beta^{*}\right)-E d_{t}\left(\beta^{*}\right)\right\}$ at frequency zero. This is somewhat a special case. If the estimator used is not MLE, or if the two density forecasts use different sets of explanatory variables, for example, estimation uncertainty becomes relevant for the asymptotic distribution, as discussed in Section 4.1. In these situations, asymptotic irrelevance of parameter estimation uncertainty can be attained by imposing that Assumption 3 holds with $\pi=0$ (see West, 1996 for a discussion), in which case the appropriate asymptotic variance will still be (13).

\subsection{Bootstrap likelihood ratio tests}

The asymptotic test can be complemented with and compared to the bootstrap test that was proposed in Section 4, with a weight function that is now identically one. For the likelihood ratio test, we also explore the possibility of improving the accuracy of the bootstrap confidence interval $C I$ in (10). We do so by use of the so-called 'bootstrap- $t$ ' approach (see, e.g., Efron and Tibshirani, 1993). The procedure deviates from the one described in Section 4 in that it requires to calculate for each artificial sample $b$, both the test statistic $L R_{n}^{b}{ }^{5}$ and an estimate $\hat{\sigma}_{n}^{b}$ of its standard deviation. The relevant standard deviation is $\sigma / \sqrt{n}$, where $\sigma$ is the square root of the asymptotic variance defined in (13), which can be estimated by kernel-based estimators, such as the Newey-West (1987) estimator. A bootstrap- $t$ confidence interval for $L R_{n}$ with $(1-\alpha) 100 \%$ nominal coverage is then computed as

$$
C I-t=\left[L R_{n}-u^{*}(1-\alpha / 2) \hat{\sigma}_{n}, L R_{n}-u^{*}(\alpha / 2) \hat{\sigma}_{n}\right]
$$

where $u^{*}(1-\alpha / 2)$ and $u^{*}(\alpha / 2)$ are the $1-\alpha / 2$ and the $\alpha / 2$ quantiles of the empirical distribution of $\left(L R_{n}^{b}-L R_{n}\right) / \hat{\sigma}_{n}^{b}, b=1, \ldots, B$ and $\hat{\sigma}_{n}$ is an estimate of the standard deviation of $L R_{n}$. A theoretical

\footnotetext{
${ }^{5} L R_{n}^{b}$ is equivalent to $W L R_{n}^{b}$ in (9) with $w(\cdot)$ identically equal to one.
} 
result (e.g., Shao and Tu, 1995) proves that the bootstrap- $t$ confidence interval (14) is more accurate than the bootstrap confidence interval (10) or the confidence interval implied by the asymptotic normal approximation in Theorem 2. ${ }^{6}$ The higher order accuracy of the bootstrap- $t$ confidence interval is an asymptotic result. In practice, its superior performance in finite samples will likely depend on the quality of the estimator for the variance. It is thus a worthwhile exercise to contrast the performance of the proposed tests in samples of the sizes typically available in practice.

\section{Monte Carlo experiment}

In this section, we analyze and compare the size and power of the likelihood ratio tests proposed in the previous section. Due to the inherent difficulties in finding plausible non-nested models that satisfy the null hypothesis, we restrict attention to the case where the two density forecasts are both normal but their specification for the conditional mean depends on a common autoregressive term and on an exogenous variable, which is different for the two densities. This situation could arise in practical applications when, for example, two economic theories postulate that different explanatory variables have predictive content for the variable of interest. Let $Y_{t}$ be the variable of interest, and $X_{1 t}$ and $X_{2 t}$ be the explanatory variables. Let $\Omega_{t}=\left\{y_{t-j}, x_{1 t+1-j}, x_{2 t+1-j} ; j \geq 0\right\}$ be the information set at time $t$. We consider the following specifications for the true conditional density and for density forecasts $f$ and $g$.

$$
\begin{aligned}
D G P & : Y_{t} \mid \Omega_{t} \sim N\left(\rho^{*} y_{t-1}+\alpha^{*} x_{1 t}+\beta^{*} x_{2 t}, 1\right) \\
\text { Forecast } 1 & : \quad Y_{t} \mid \Omega_{t} \sim N\left(\rho y_{t-1}+\alpha x_{1 t}, 1\right) \equiv f \\
\text { Forecast } 2 & : \quad Y_{t} \mid \Omega_{t} \sim N\left(\rho y_{t-1}+\beta x_{2 t}, 1\right) \equiv g
\end{aligned}
$$

The variables $X_{1 t}$ and $X_{2 t}$ are independent $N(0,1)$ random variables. Notice that we assumed the true variance to be known by both forecasters. This simplification, although perhaps unrealistic, was needed to obtain a parameterization for the DGP such that $f$ and $g$ satisfy the null hypothesis. In this case, one can easily show that the probability limits for the parameters of $f$ and $g$ are respectively $\left(\rho^{*}, \alpha^{*}\right)^{\prime}$ and $\left(\rho^{*}, \beta^{*}\right)^{\prime}$ and that the expected difference of the scores is $L R^{*}=\left(\alpha^{*}\right)^{2}-\left(\beta^{*}\right)^{2}$. A parameterization for the DGP such that $\alpha^{*}=\beta^{*}$ will thus satisfy the null hypothesis, and it will be used to investigate the size of the tests. The parameterization used for the size study is $\left(\rho^{*}, \alpha^{*}, \beta^{*}\right)=$ $(.5, .1, .1)$. The power curve is obtained by keeping $\alpha^{*}=.1$ fixed and increasing $\beta^{*}$. The number of

\footnotetext{
${ }^{6}$ Higher accuracy of a confidence interval means that its coverage level is closer to the nominal level $(1-\alpha) 100 \%$.
} 
Monte Carlo iterations is chosen to be 1000 . We consider a total of 15 different combinations of insample $(R)$ and out-of-sample $(n)$ sizes: $R=50,100,150$ and $n=25,50,75,100,150$. This range and relative proportion of in-sample and out-of-sample sizes seems broad enough to represent the typical situation of macroeconomic forecasting. To represent the larger sample sizes that may arise in some financial applications, we further consider the two pairs $(R, n)=(500,250)$ and $(R, n)=(650,350)$.

For each iteration, the parameters of $f$ and $g$ are estimated by ML on the first sample of size $R$. The density forecasts for period $R+1$ are then formed as $f: N\left(\hat{\rho}_{R} y_{R}+\hat{\alpha}_{R} x_{1 R+1}, 1\right)$ and $g$ : $N\left(\hat{\rho}_{R} y_{R}+\hat{\beta}_{R} x_{2 R+1}, 1\right)$. Each density is evaluated at the realized value for the variable $y_{R+1}$ and the first observed score difference is obtained as $d_{R+1}=\log f\left(y_{R+1} \mid \Omega_{R} ; \hat{\rho}_{R}, \hat{\alpha}_{R}\right)-\log g\left(y_{R+1} \mid \Omega_{R} ; \hat{\rho}_{R}, \hat{\beta}_{R}\right)$. The sample is then augmented by including observation $y_{R+1}$ and the procedure is repeated on the sample of size $R+1$ to obtain the score difference $d_{R+2}$, and so forth. The recursion generates a total of $n$ score differences $d_{R+1}, \ldots, d_{R+n}$ that are averaged to obtain $L R_{n}$ as in (12). The three likelihood ratio tests proposed in Sections 5 are then performed and their rejection frequencies calculated over the Monte Carlo iterations. We refer to the three tests as 'asymptotic LR test', 'bootstrap LR test' and 'bootstrap-t LR test'. As noted in Section 5.1, when the forecasts are conditional on different information sets the asymptotic variance is (13) as long as Assumption 3 holds with $\pi=0$. We implicitly impose this requirement, and use (13) in the computations for the asymptotic LR test. The impact of ignoring estimation uncertainty will emerge from the analysis of the power curves for decreasing $\pi$. Table 1 reports the empirical size of the three tests for nominal size .05 .

\section{[TABLE 1 HERE]}

The asymptotic LR test is oversized for an out-of-sample size $n \leq 50$. A mild tendency to overreject is still present for the two bootstrap tests when the size of the out-of-sample is small, but they are overall better sized than the asymptotic test for all combinations of $R$ and $n$. All tests have good size for an out-of-sample $n \geq 75$.

Figures 2-5 show the power curves for a selection of in-sample and out-of-sample pairs.

\section{[FIGURES 2-5 HERE]}

On the horizontal axis, instead of reporting the increasing distance between the values of the coefficients $\alpha^{*}$ and $\beta^{*}$, we choose to report the corresponding difference in $R^{2}$ from the regressions that define density forecasts $f$ and $g .^{7}$ For the size study we let $\alpha^{*}=\beta^{*}=0.1$, which implies an

\footnotetext{
${ }^{7}$ The $R^{2}$ from each regression can be shown to equal $R_{f}^{2}=1-\left(1-\rho^{* 2}\right)\left(\beta^{* 2}+1\right) /\left(\alpha^{* 2}+\beta^{* 2}+1\right)$ and $R_{g}^{2}=$ $1-\left(1-\rho^{* 2}\right)\left(\alpha^{* 2}+1\right) /\left(\alpha^{* 2}+\beta^{* 2}+1\right)$ and thus the difference is given by $R_{f}^{2}-R_{g}^{2}=\left(1-\rho^{* 2}\right)\left(\alpha^{* 2}-\beta^{* 2}\right) /\left(\alpha^{* 2}+\beta^{* 2}+1\right)$
} 
equal $R^{2}$ for the two models of 0.36 , and for the power study we let $\beta^{*}$ increase, so that the difference in $R^{2}$ for the two forecasts varies between 0 and 0.5 . The graphs are presented in order of increasing out-of-sample size. A pattern that emerges form the figures is that the three tests have different power when the out-of-sample size $n$ is small, but the power tends to become equal across the three tests for larger $n$. For $n=25$ (Figure 2), the asymptotic LR test has higher power than the bootstrap tests, at the price of high size distortions. For the same value of $n$, the bootstrap-t LR test has slightly lower power than the bootstrap LR test, but this divergence in power between the bootstrap tests disappears for $n \geq 75$. Notice also that all power curves become steeper as the out-of-sample size increases, a sign of the consistency of the tests. A final conclusion that emerges from the comparison of the two panels in each figure is that the size of the in-sample seems not to affect the properties of the tests. The size and power differences seem to be driven only by the size of the out-of-sample. This is a hopeful indication that imposing the condition that the in-sample size grows faster than the out-of-sample (i.e., Assumption 3 with $\pi=0$ ) does not affect the properties of the tests, while considerably simplifying the estimation of the asymptotic variance of the likelihood ratio test. This in turn would suggest that ignoring parameter estimation uncertainty does not significantly alter the properties of the likelihood ratio tests in our framework.

\section{Empirical application}

In this section, the tests proposed in the paper are utilized to compare one-step-ahead univariate density forecasts for the S\&P500 index obtained from GARCH models with different distributional assumptions. The data are daily U.S. returns on the S\&P500 from 1/1/1990 to 8/3/2001 obtained from Datastream. The return series is derived from the price index data, $p_{t}$, as $y_{t}=100 \log \left(p_{t} / p_{t-1}\right)$, so that $y_{t}$ represents the continuously compounded return (in percent) on the index. We model the returns as a $\operatorname{GARCH}(1,1)$

$$
\begin{aligned}
y_{t} & =\mu+\varepsilon_{t}, \\
\varepsilon_{t} \mid \Omega_{t-1} & \sim f\left(0, \sigma_{t}\right), \\
\sigma_{t}^{2} & =w+\alpha \varepsilon_{t-1}^{2}+\beta \sigma_{t-1}^{2} .
\end{aligned}
$$

This model specification will generate density forecasts with time-varying variance and functional form specified by $f$. When the model (16) is used for prediction, the accuracy of the forecast critically depends on the distribution chosen for the standardized residuals. It is widely acknowledged that a 
normality assumption for the conditional distribution of the disturbances $\varepsilon_{t}$ does not account for the excess kurtosis that characterizes the residuals from a fitted GARCH model ${ }^{8}$. A way to incorporate excess kurtosis in the model is the use of fat-tailed distributions for the disturbances. Popular examples are the Student's $t$ (proposed by Bollerslev, 1987) and the generalized error distribution (GED), utilized by Nelson (1991). A further characteristic that one might want to account for is the possible skewness of returns, that can be captured, for example, by a skewed- $t$ distribution (see Hansen, 1994). In the following, we will refer to density forecasts generated by model (16) with the three different specifications for the disturbances as $t-\mathrm{GARCH}, G E D-\mathrm{GARCH}$ and skewt-GARCH. The goal is to do pairwise comparisons to select the model that yields the most accurate density forecasts, and then isolate the regions of the distribution where such outperformance takes place. We are able to apply the tests proposed in the paper to the non-nested pairs ( $t-\mathrm{GARCH}, G E D-\mathrm{GARCH})$ and ( skewt-GARCH, GED-GARCH). The comparison of the out-of-sample performance of $t-\mathrm{GARCH}$ and skewt-GARCH is not possible due to the nestedness of the two models.

We proceed as follows. We divide the available sample of $T=2928$ observations in two parts, using the first $R$ data for estimation and leaving the remaining $n$ observations for out-of-sample evaluation. We consider a range of sizes for $n$ that varies between $n=100$ (and thus $R=2828$ ) and $n=1500$ (which corresponds to $R=1428$ ), using increments of $100 \mathrm{in} n$. The first set of density forecasts is obtained by estimating the parameters of (16) for each of the three distributional assumptions on the sample of size $R$. This involves estimating the unconditional mean $\hat{\mu}$, the GARCH parameters $(\hat{w}, \hat{\alpha}, \hat{\beta})$ and the shape parameters of each distribution by ML. The $t-\mathrm{GARCH}$ density forecast for the variable $Y_{R+1}$ is, for example, a Student's $t$ with mean $\hat{\mu}$, variance $\sigma_{R+1}^{2}=\hat{w}+\hat{\alpha} \varepsilon_{R}^{2}+\hat{\beta} \sigma_{R}^{2}$ and shape parameter as estimated on the sample of size $R$. For each of the three density forecasts, we then evaluate the $\log$ of the density at the realized value of $y_{R+1}$. This generates the first set of three logarithmic scores. The procedure is then repeated on the samples of sizes $R+1, \ldots, T-1$, and it yields a sequence of $n$ logarithmic scores.

Before proceeding with the tests, we consider the sequence of volatility forecasts implied by the three different models, that are plotted in Figure 6 for the case of $n=1500$. As the reader can see, the volatility forecasts implied by the different distributional assumptions are virtually indistinguishable. As a consequence, any approach to model selection that relies on the comparison on volatility forecast accuracy is likely doomed to fail.

\footnotetext{
${ }^{8}$ Evidence of non-normality of financial assets' returns has been documented, inter alia, by Mandelbrot (1963), Fama (1965), Bollerslev (1987).
} 
[FIGURE 6 HERE]

In contrast, the tests proposed in the paper will help detect any superior predictive ability that is solely due to the different distributional assumptions of the three models. The first set of results compares the accuracy of $t-\mathrm{GARCH}$ and $G E D-\mathrm{GARCH}$ density forecasts.

\section{[FIGURES 7-9 HERE]}

The $L R_{n}$ statistic is computed by letting $f$ be the $G E D$ density and $g$ the Student's $t$ density. A value of $L R_{n}$ that is significantly less than zero will thus mean that the $t$-GARCH density forecasts outperform the GED-GARCH forecasts. Figure 7 plots the p-values of the asymptotic LR test for increasing out-of-sample size $n$. Equal performance of the two density forecasts is rejected at a $95 \%$ confidence level for values of $n$ greater than 1000. For these sample sizes, the $L R_{n}$ is negative (Figure 8), indicating that $t-\mathrm{GARCH}$ forecasts are more accurate than $G E D-\mathrm{GARCH}$ forecasts. This conclusion is confirmed by the bootstrap-t LR test, reported in Figure 8. The figure shows the value of $L R_{n}$ for different $n$ and the relative bootstrap 95\% confidence interval. For values of $n$ greater than $1000, L R_{n}$ is significantly negative.

To detect in what regions of the unconditional distribution of the returns the $t$-GARCH density forecasts outperform the $G E D-\mathrm{GARCH}$ forecasts, we consider the weighted likelihood ratio tests for the four weight functions shown in Figure 1. The four panels in Figure 9 plot the values of the weighted likelihood ratio statistic $W L R_{n}$ for the different weight functions, together with $95 \%$ bootstrap confidence intervals. The value of $W L R_{n}$ is significantly negative for the weight function $w_{1}$, which represents the center of the unconditional distribution. This indicates that the $t-\mathrm{GARCH}$ density forecasts significantly outperform the $G E D-\mathrm{GARCH}$ forecasts for values of returns that fall near the center of the unconditional distribution. In other words, $t-\mathrm{GARCH}$ density forecasts are better than $G E D-\mathrm{GARCH}$ forecasts at predicting returns in 'normal' days, while the two densities are equally accurate in predicting 'extreme' events.

We then investigate whether allowing the best model $(t-\mathrm{GARCH})$ to incorporate skewness will lead to further improvements in its performance relative to the $G E D-\mathrm{GARCH}$ forecast model. The second set of results compares the performance of skewt-GARCH and GED-GARCH density forecasts. Figures 10-12 are the equivalent of Figures 7-9 for the new pair of density forecasts.

[FIGURES 10-12 HERE] 
As expected, given the nestedness of skewt and $t$ distributions, Figures 10 and 11 lead to the same conclusion highlighted by Figures 7 and 8 . The skewt-GARCH density forecasts outperform the GED-GARCH forecasts. Figure 12 reveals that the skewt-GARCH outperforms the GED-GARCH in both the center and the right tail of the unconditional distribution of returns. That is, the skewt-GARCH is better at predicting returns in normal days and in days when returns are relatively high (the definition of what constitutes a normal day and a high return is necessarily imprecise, given the arbitrary choice of weight function). In conclusion, a $t-\mathrm{GARCH}$ or skewt-GARCH density forecasts are seen to be more accurate than a $G E D-\mathrm{GARCH}$ density forecast. A similar finding has been documented by Bollerslev, Engle and Nelson (1994) in a non-predictive setting.

\section{Conclusion}

The paper proposed a number of tests that can be used to compare conditional density forecasts, or in the more general context of model selection based on out-of-sample forecasting performance. The tests can be utilized in both a univariate and a multivariate setting, even though in the paper we focused for simplicity on the univariate case. We restricted attention to the case of density forecasts derived from conditional parametric models that are non-nested, with known or estimated parameters.

We considered measuring the performance of the density forecasts by so-called 'scoring rules', which are loss functions defined over the probability forecast and the outcome of the variable. In particular, we restricted attention to the logarithmic scoring rule, and suggested ranking the forecasts according to the relative magnitude of a weighted average of the scores measured over the available sample, which led to the development of 'weighted likelihood ratio' tests. We showed how these tests can be utilized to isolate the performance of competing density forecasts in different regions of the unconditional distribution of the variable of interest. Loosely speaking, the tests can help distinguish, for example, the relative performance of the density forecasts in 'normal' days from the performance in days when the variable takes on 'extreme' values. We proposed both asymptotic and bootstrap weighted likelihood ratio tests. As a special case of a weighted likelihood ratio test, we obtained an extension of Vuong's (1989) likelihood ratio test for non-nested hypotheses to time series data and out-of-sample testing. When the parameters of the density forecasts are estimated by maximum likelihood, we further pointed out that the asymptotic likelihood ratio test becomes particularly easy to compute. The performance of the asymptotic and bootstrap likelihood ratio 
tests in finite samples was analyzed through a Monte Carlo simulation that considered the case where the alternative density forecasts have the same functional form, but use different exogenous variables in their conditional mean specifications. We found the asymptotic likelihood ratio test to be moderately oversized for very small out-of-sample sizes, while the bootstrap tests had good size and power for all combinations of in-sample and out-of-sample sizes. The tests proposed in the paper were finally used in an empirical application comparing density forecasts obtained from GARCH-type models with different distributional assumptions for the standardized residuals. The data considered were the series of S\&P 500 daily returns and the models used for constructing density forecast were GARCH(1,1) with Student's $t$, generalized error distribution $(G E D)$ and skewed Student's $t$ (skewt) disturbances. We concluded that density forecasts from a $t-\mathrm{GARCH}$ and from a skewt-GARCH models are more accurate than density forecasts from a GED-GARCH model, and the superior performance was seen to occur in 'normal days'. The skewt-GARCH forecasts also outperformed the GED-GARCH forecasts on days when the returns on the S\&P 500 are large and positive. 


\section{Proofs}

Proof of Theorem 1. Since $W L R_{n}=\iota n^{-1} \sum_{t=R}^{T-1} q_{t+1}\left(\hat{\beta}_{t}\right)$ and $W L R^{*}=\iota E q_{t+1}\left(\beta^{*}\right)$, we have that $\sqrt{n}\left(W L R_{n}-W L R^{*}\right)=\iota \sqrt{n}\left[n^{-1} \sum_{t=R}^{T-1} q_{t+1}\left(\hat{\beta}_{t}\right)-E q_{t+1}\left(\beta^{*}\right)\right]$. We will make use of Theorem 4.1 of West (1996) to show that

$$
\sqrt{n}\left[n^{-1} \sum_{t=R}^{T-1} q_{t+1}\left(\hat{\beta}_{t}\right)-E q_{t+1}\left(\beta^{*}\right)\right] \stackrel{D}{\rightarrow} N(0, \Sigma),
$$

from which it follows that $\sqrt{n}\left(W L R_{n}-W L R^{*}\right) \stackrel{D}{\rightarrow} N\left(0, \iota \Sigma \iota^{\prime}\right)$. To be able to apply Theorem 4.1, we must first show that Assumptions 1-4 of West (1996) (which we will call W1-W4) are satisfied by the vector $q_{t+1}(\beta)$.

W1-(a) requires $q_{t+1}(\beta)$ to be measurable and twice continuously differentiable in a neighborhood of $\beta^{*}$, which is implied by Assumption 1-(a).

W1-(b) requires the matrix $\nabla_{\beta}^{2} q_{t+1}(\beta)=\left(\begin{array}{cc}\nabla_{\gamma}^{2} \log g\left(Y_{t+1} \mid \Omega_{t}, \gamma\right) & 0 \\ 0 & \nabla_{\theta}^{2} \log f\left(Y_{t+1} \mid \Omega_{t}, \theta\right)\end{array}\right)$ to be bounded by a variable with finite expectation, which follows from the boundedness of the two diagonal components imposed by Assumption 1-(b).

W2 assumes that the parameter estimates $\hat{\beta}_{t}$ can be written as $\hat{\beta}_{t}-\beta^{*}=B(t) A(t)$, where $B(t) \stackrel{a . s .}{\rightarrow} B, B$ a matrix of rank $k=k_{1}+k_{2}$, and $A(t)=t^{-1} \sum_{s=1}^{t} a_{s}\left(\beta^{*}\right)$, with $E a_{s}\left(\beta^{*}\right)=0$. This follows directly from Assumption 1-(c), by letting $B(t) \equiv\left(\begin{array}{cc}B^{g}(t) & 0 \\ 0 & B^{f}(t)\end{array}\right)$ and $B$ and $a_{t}\left(\beta^{*}\right)$ to be as defined in (8).

W3-(a) imposes a bound on the fourth moments of $a_{t}\left(\beta^{*}\right), q_{t+1}\left(\beta^{*}\right)$ and $\nabla_{\beta} q_{t+1}\left(\beta^{*}\right)$. Existence of the fourth moments of $a_{t}\left(\beta^{*}\right)$ is directly implied by Assumption 1-(d). The boundedness on the fourth moments of $q_{t+1}\left(\beta^{*}\right)$ and $\nabla_{\beta} q_{t+1}\left(\beta^{*}\right)$ is implied by the existence of the fourth moments of each component of the two vectors. For illustration, we only prove this claim for the second component of $q_{t+1}\left(\beta^{*}\right)$, which equals $w\left(Y_{t+1}\right) \log f\left(Y_{t+1} \mid \Omega_{t}, \theta^{*}\right)$. From Assumption 1-(d), it follows that there exists a $d>1$ such that $E\left|\log f\left(Y_{t+1} \mid \Omega_{t}, \theta^{*}\right)\right|^{4 d}<\infty$. Consider $E\left|w\left(Y_{t+1}\right) \log f\left(Y_{t+1} \mid \Omega_{t}, \theta^{*}\right)\right|^{4 d^{\prime}}$, with $d^{\prime}=\frac{d}{1+\varepsilon}$, for some $\varepsilon>0$. Since $w\left(Y_{t+1}\right) \geq 0$, and by applying Hölder's inequality, we have

$$
\begin{gathered}
E\left|w\left(Y_{t+1}\right) \log f\left(Y_{t+1} \mid \Omega_{t}, \theta^{*}\right)\right|^{4 d^{\prime}}=\left.E\left|w\left(Y_{t+1}\right)^{4 d^{\prime}}\right| \log f\left(Y_{t+1} \mid \Omega_{t}, \theta^{*}\right)\right|^{4 d^{\prime}}|| \\
\leq\left(E\left|w\left(Y_{t+1}\right)^{4 d^{\prime}}\right|^{\frac{1+\varepsilon}{\varepsilon}}\right)^{\frac{\varepsilon}{1+\varepsilon}}\left(E\left|\log f\left(Y_{t+1} \mid \Omega_{t}, \theta^{*}\right)\right|^{4 d^{\prime}(1+\varepsilon)}\right)^{\frac{1}{1+\varepsilon}} \\
=\left(E\left|w\left(Y_{t+1}\right)\right|^{\frac{4 d}{\varepsilon}}\right)^{\frac{\varepsilon}{1+\varepsilon}}\left(E\left|\log f\left(Y_{t+1} \mid \Omega_{t}, \theta^{*}\right)\right|^{4 d}\right)^{\frac{1}{1+\varepsilon}}<\infty,
\end{gathered}
$$


since the first term is finite because of boundedness of $w(\cdot)$, and the second term is bounded by Assumption 1-(d).

W3-(b) assumes the vector $\left[q_{t+1}\left(\beta^{*}\right)^{\prime}, \operatorname{vec}\left(\nabla_{\beta} q_{t+1}\left(\beta^{*}\right)\right)^{\prime}, a_{t}\left(\beta^{*}\right)^{\prime}\right]^{\prime}$ to be strong mixing of size $-3 d /(d-$ 1). This follows from $\left\{Y_{t}\right\}$ being strong mixing of size $-3 d /(d-1)$ by Assumption 1-(e), and from Lemma 2.1 of White and Domowitz (1984), showing that measurable functions of mixing processes are mixing of the same size.

W3-(c) requires $\left[q_{t+1}\left(\beta^{*}\right)^{\prime}, \operatorname{vec}\left(\nabla_{\beta} q_{t+1}\left(\beta^{*}\right)\right)^{\prime}, a_{t}\left(\beta^{*}\right)^{\prime}\right]^{\prime}$ to be covariance stationary, which is directly implied by Assumption 1-(f).

W3-(d) assumes $S_{q q}$ defined in (8) to be positive definite. This is ensured by Assumption 2, requiring the two components of $q_{t+1}\left(\beta^{*}\right)$ to be distinct.

W4 coincides with Assumption 3.

We can thus apply Theorem 4.1 of West (1996) to prove (17).

The second part of the theorem follows directly from the fact that $\sqrt{n}\left(W L R_{n}-W L R^{*}\right) \stackrel{D}{\rightarrow}$ $N\left(0, \sigma^{2}\right)$, with $\sigma^{2}>0$ due to positive definiteness of $\Sigma$, and from Slutsky's Theorem.

Proof of Theorem 2. When $w(y)=1$, for all $x$, and the parameter estimates are QMLEs, it follows that $F \equiv E\left(\begin{array}{cc}\nabla_{\gamma} \log g\left(Y_{t+1} \mid \Omega_{t} ; \gamma^{*}\right) & 0 \\ 0 & \nabla_{\theta} \log f\left(Y_{t+1} \mid \Omega_{t} ; \theta^{*}\right)\end{array}\right)=\mathbf{0}$. In this case, thus, the matrix $\Sigma$ defined in (8) reduces to $S_{q q}$, and the asymptotic variance of the likelihood ratio test becomes $\sigma^{2}=\iota S_{q q} \iota^{\prime}=\sum_{j=-\infty}^{+\infty} \iota \delta_{q q}(j) \iota^{\prime}=\sum_{j=-\infty}^{+\infty} \delta_{d d}(j)$. 


\section{References}

[1] Andrews, D. W. K. (1991): 'Heteroskedasticity and Autocorrelation Consistent Covariance Matrix Estimation', Econometrica, 59, 817-858.

[2] Artzner, P., Delbaen, F., Eber, J. M., Heath, D. (1997): 'Thinking Coherently', Risk, 10, 68-71.

[3] Berkowitz, J. (2000): 'The Accuracy of Density Forecasts in Risk Management', forthcoming Journal of Business and Economic Statistics.

[4] Blattenberger, G., Lad, F. (1988): 'An Application of Operational-Subjective Statistical Methods to Rational Expectations', Journal of Business and Economic Statistics, 6, 453-464.

[5] Bollerslev, T. (1987): 'A Conditional Heteroskedastic Time Series Model for Speculative Prices and Rates of Return', Review of Economics and Statistics, 69, 542-547.

[6] Bollerslev, T., Engle, R. F., Nelson, D. B. (1994): 'ARCH Models', in Handbook of Econometrics, vol. 4, Chapter 49, Engle, R. F. and McFadden, D. (eds.), Elsevier Science B. V., Amsterdam, The Netherlands.

[7] Christoffersen P. F., Diebold, F. X. (1997): 'Optimal Prediction under Asymmetric Loss', Econometric Theory, 13, 808-817.

[8] Clements, M. P., Smith, J. (2000): 'Evaluating the Forecast Densities of Linear and Nonlinear Models: Applications to Output Growth and Unemployment', Journal of Forecasting, 19, 255-276.

[9] Diebold, F. X., Gunther, T. A., Tay, A. S. (1998): 'Evaluating Density Forecasts with Applications to Financial Risk Management', International Economic Review, 39, 863-883.

[10] Diebold, F. X., Lopez, J. A. (1996): 'Forecast Evaluation and Combination', in Handbook of Statistics, Volume 14: Statistical Methods in Finance, G. S. Maddala, C. R. Rao (eds.). Amsterdam: North-Holland, 241-268.

[11] Diebold, F. X., Mariano, R. S. (1995): 'Comparing Predictive Accuracy', Journal of Business and Economic Statistics, 13, 253-263.

[12] Diebold, F. X., Tay, A.S., Wallis, K. F. (1999): "Evaluating Density Forecasts of Inflation: The Survey of Professional Forecasters", in R. Engle and H. White (eds.), Festschrift in Honour of C.W.J. Granger, 76-90, Oxford University Press. 
[13] Duffie, D., Pan, J. (1996): 'An Overview of Value at Risk', Journal of Derivatives, 4, 13-32.

[14] Fama, E. F. (1965): 'The Behavior of Stock-Market Prices', Journal of Business 38, 34-105.

[15] Hansen, B. (1994): ‘Autoregressive Conditional Density Estimation', International Economic Review, 35, 705-730.

[16] Hong, Y. (2000): 'Evaluation of Out-of-Sample Density Forecasts with Applications to S\&P 500 Stock Prices', manuscript.

[17] Hong, Y., White, H. (2000): 'Asymptotic Distribution Theory for Nonparametric Entropy Measures of Serial Dependence', manuscript.

[18] Künsch, H. R. (1989): 'The Jackknife and the Bootstrap for General Stationary Observations', Annals of Statistics, 17, 1217-1241

[19] Liu, R. Y., Singh, K. (1992): 'Moving Blocks Jackknife and Bootstrap Capture Weak Dependence' in Exploring the Limits of the Bootstrap, R. LePage and L. Billiard (eds), Wiley, New York

[20] Lopez, J. A. (2001): 'Evaluating the Predictive Accuracy of Volatility Models', Journal of Forecasting, 20, 87-109.

[21] Mandelbrot, B. (1963): 'The Variation of Certain Speculative Prices', Journal of Business, 26, 395-419.

[22] Matheson, J. E., Winkler, R. L. (1976): 'Scoring Rules for Continuous Probability Distributions', Management Science, 22, 1087-1096.

[23] McCracken, M. W. (2000): 'Robust out-of-sample Inference', Journal of Econometrics, 99, 195223.

[24] Nelson, D. (1991): 'Conditional Heteroskedasticity in Asset Returns: A New Approach', Econometrica, 59, 347-370.

[25] Newey, W. K., West, K. D. (1987): 'A Simple, Positive Semidefinite, Heteroskedasticity and Autocorrelation Consistent Covariance Matrix", Econometrica, 55, 703-708.

[26] Patton, A. J. (2001): 'Modelling Time-Varying Exchange Rate Dependence using the Conditional Copula', UCSD working paper n. 2001-09 
[27] Politis, D., Romano, J. (1994): 'The Stationary Bootstrap', Journal of the American Statistical Association, 89, 1303-1313

[28] Rivers, D., Vuong, Q. (1999): 'Model Selection Tests for Nonlinear Dynamic Models ', University of Southern California manuscript

[29] Shao, J., Tu, D. (1995): The Jackknife and Bootstrap, Springer-Verlag, New York

[30] Savage, L. J. (1971): 'Elicitation of Personal Probabilities and Expectations', Journal of the American Statistical Association, 66, 783-801.

[31] Soderlind, P., Svensson, L. (1997): 'New Techniques to Extract Market Expectations from Financial Instruments', Journal of Monetary Economics, 40, 383-429.

[32] Tay, A. S., Wallis, K. F. (2000): 'Density Forecasting: A Survey', Journal of Forecasting, 19, $235-254$.

[33] Vuong, Q. H. (1989): 'Likelihood Ratio Tests for Model Selection and Non-nested Hypotheses', Econometrica, 57, 307-333.

[34] Weigend, A. S., Shi, S. (2000): 'Predicting Daily Probability Distributions of S\&P500 Returns', Journal of Forecasting, 19, 375-392.

[35] Weiss, A. A. (1996): 'Estimating Time Series Models Using the Relevant Cost Function', Journal of Applied Econometrics, 11, 539-560.

[36] West, K. D. (1996): 'Asymptotic Inference about Predictive Ability', Econometrica, 64, 10671084 .

[37] White, H. (1996): Estimation, Inference and Specification Analysis, Cambridge University Press.

[38] White, H. (2000): 'A Reality Check for Data Snooping', Econometrica, 68, 1097-1126.

[39] White, H., Domowitz, I. (1984): 'Nonlinear Regression with Dependent Observations', Econometrica, 52, 143-162.

[40] Winkler, R. L. (1967): 'The Quantification of Judgement: Some Methodological Suggestions', Journal of the American Statistical Association, 62, 1105-1120. 


\section{Table 1}

Size of nominal .05 tests

A. LR asymptotic test

\begin{tabular}{cccccc}
\hline & \multicolumn{5}{c}{$\mathrm{n}$} \\
\cline { 2 - 6 } $\mathrm{R}$ & 25 & 50 & 75 & 100 & 150 \\
\hline 50 & 0.162 & 0.095 & 0.059 & 0.042 & 0.047 \\
100 & 0.158 & 0.160 & 0.076 & 0.078 & 0.064 \\
150 & 0.197 & 0.167 & 0.086 & 0.053 & 0.065
\end{tabular}

B. LR bootstrap test

\begin{tabular}{cccccc}
\hline & \multicolumn{5}{c}{$\mathrm{n}$} \\
\cline { 2 - 6 } $\mathrm{R}$ & 25 & 50 & 75 & 100 & 150 \\
\hline 50 & 0.059 & 0.058 & 0.037 & 0.033 & 0.033 \\
100 & 0.072 & 0.065 & 0.047 & 0.052 & 0.050 \\
150 & 0.092 & 0.073 & 0.052 & 0.036 & 0.054 \\
& & C. $\mathbf{~ L R}$ bootstrap-t test & & \\
\hline & & & $\mathrm{n}$ & & \\
$\mathrm{n}$ & 25 & 50 & 75 & 100 & 150 \\
\hline 50 & 0.080 & 0.063 & 0.044 & 0.047 & 0.046 \\
100 & 0.066 & 0.073 & 0.058 & 0.069 & 0.055 \\
150 & 0.094 & 0.077 & 0.064 & 0.048 & 0.061 \\
\hline
\end{tabular}

Notes: Each panel reports the empirical size of the three likelihood ratio tests discussed in Section 5. Entries represent the rejection frequencies over 1000 Monte Carlo replications of the null hypothesis $H_{0}: E\left[\log g\left(Y_{t+1} \mid \Omega_{t} ; \gamma^{*}\right)-\log f\left(Y_{t+1} \mid \Omega_{t} ; \theta^{*}\right)\right]=0$, where the density forecasts $f, g$ and the DGP are defined in (15). The nominal size is .05. Each cell corresponds to a pair of in-sample and out-of-sample sizes $(R, n)$. 

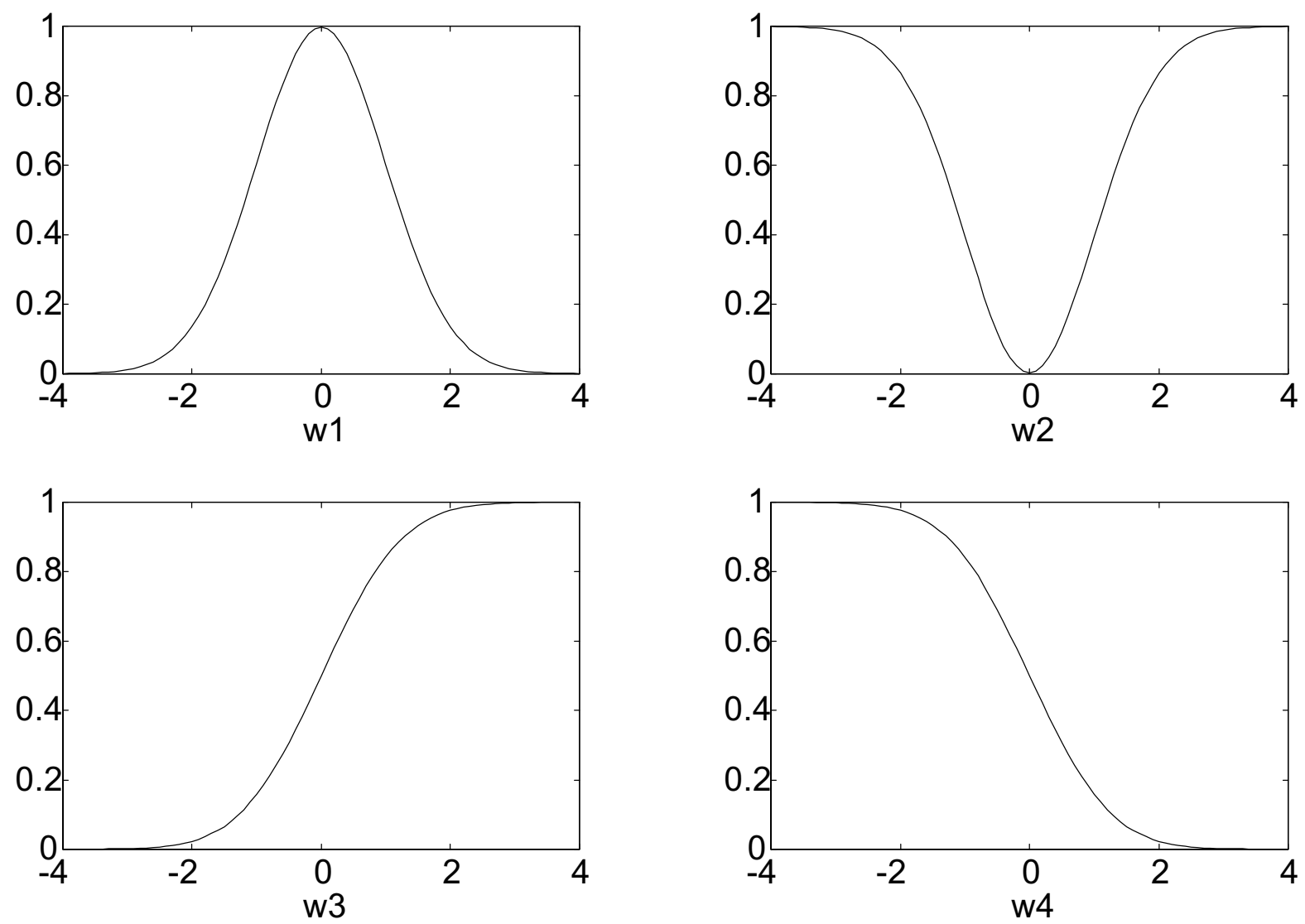

Figure 1: Weight functions for the weighted likelihood ratio test. $w_{1}(y)=\phi(y), w_{2}(y)=1-2.5 \phi(y)$, $w_{3}(y)=\Phi(y), w_{4}(y)=1-\Phi(y)$, where $\phi$ and $\Phi$ are, respectively, the standard normal pdf and cdf. 

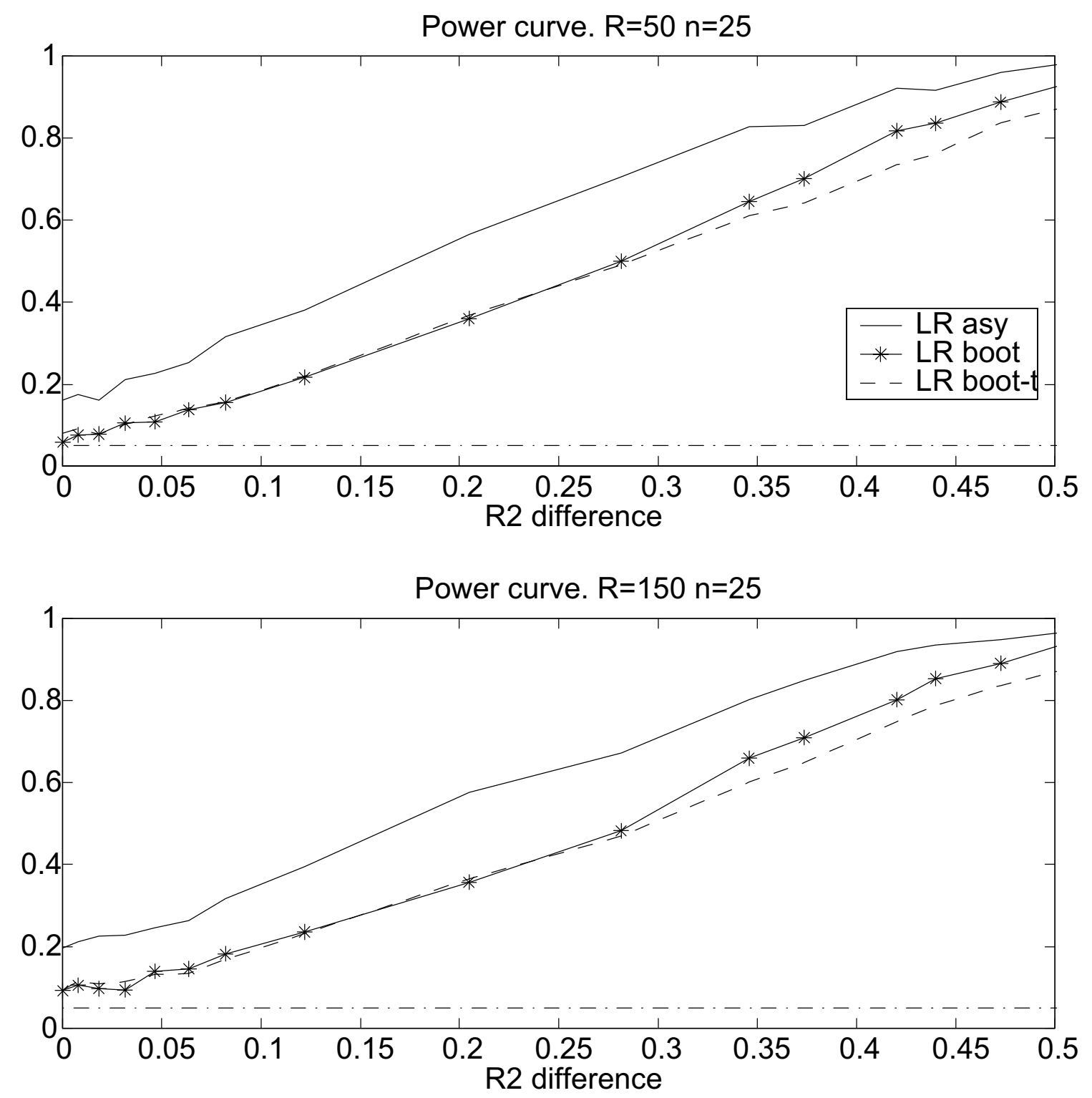

Figure 2: Power curves of LR asymptotic test, LR bootstrap test and LR bootstrap-t test in the Monte Carlo experiment discussed in Section 6. Each curve represents the rejection frequencies over 1000 Monte Carlo replications of the null hypothesis $H_{0}: E\left[\log g\left(Y_{t+1} \mid \Omega_{t} ; \gamma^{*}\right)-\log f\left(Y_{t+1} \mid \Omega_{t} ; \theta^{*}\right)\right]=0$, where the density forecasts $f, g$ and the DGP are defined in (15). The horizontal axis shows the difference in $R^{2}$ from the regressions defining the two density forecasts $f$ and $g$. Both figures consider an out-of-sample size of $n=25$. The upper panel is for in-sample size $R=50$ and the lower panel for $R=150$. 
Power curve. $R=50 n=75$
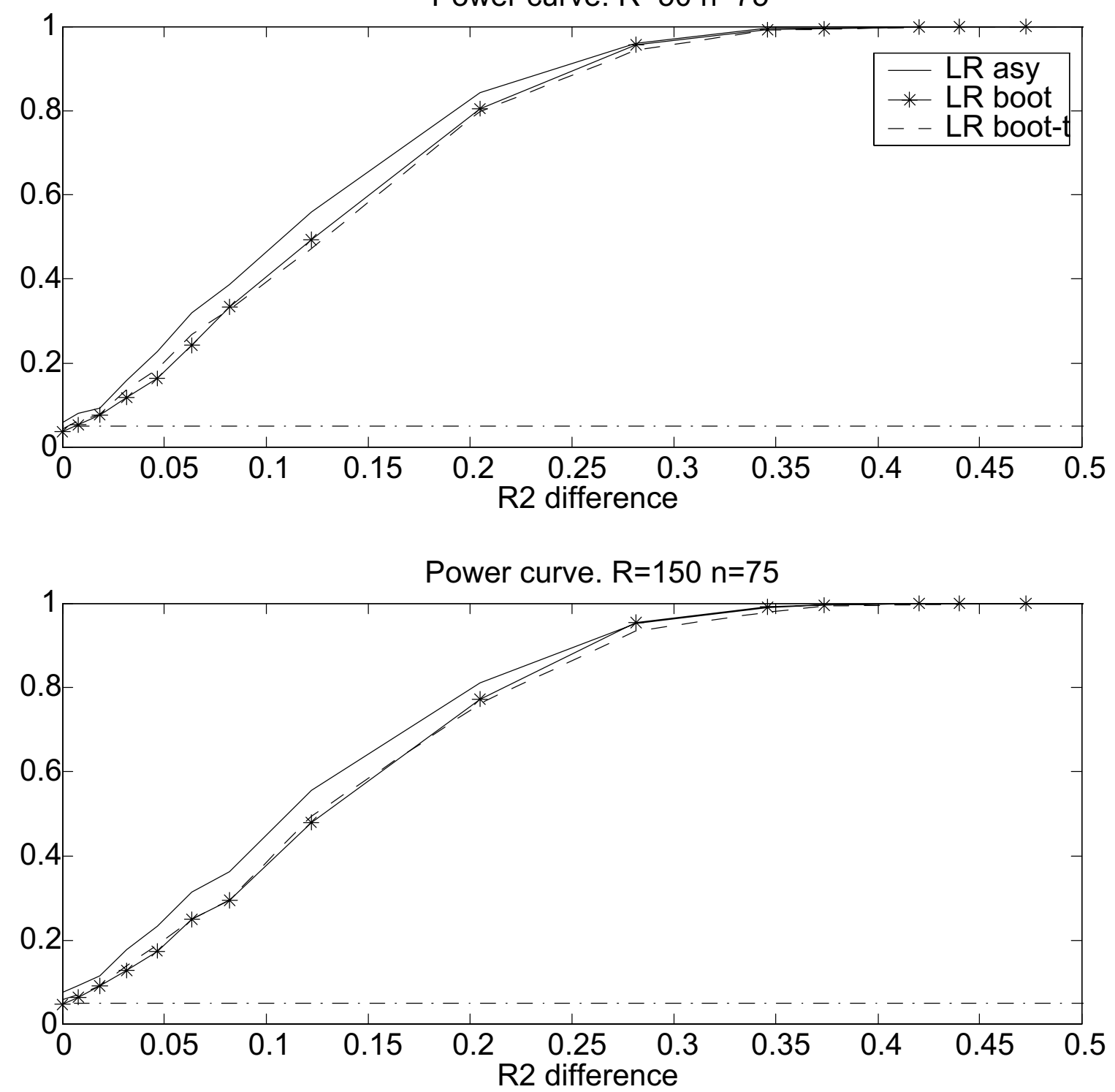

Figure 3: Power curves of LR asymptotic test, LR bootstrap test and LR bootstrap-t test in the Monte Carlo experiment discussed in Section 6. Each curve represents the rejection frequencies over 1000 Monte Carlo replications of the null hypothesis $H_{0}: E\left[\log g\left(Y_{t+1} \mid \Omega_{t} ; \gamma^{*}\right)-\log f\left(Y_{t+1} \mid \Omega_{t} ; \theta^{*}\right)\right]=0$, where the density forecasts $f, g$ and the DGP are defined in (15). The horizontal axis shows the difference in $R^{2}$ from the regressions defining the two density forecasts $f$ and $g$. Both figures consider an out-of-sample size of $n=75$. The upper panel is for in-sample size $R=50$ and the lower panel for $R=150$. 

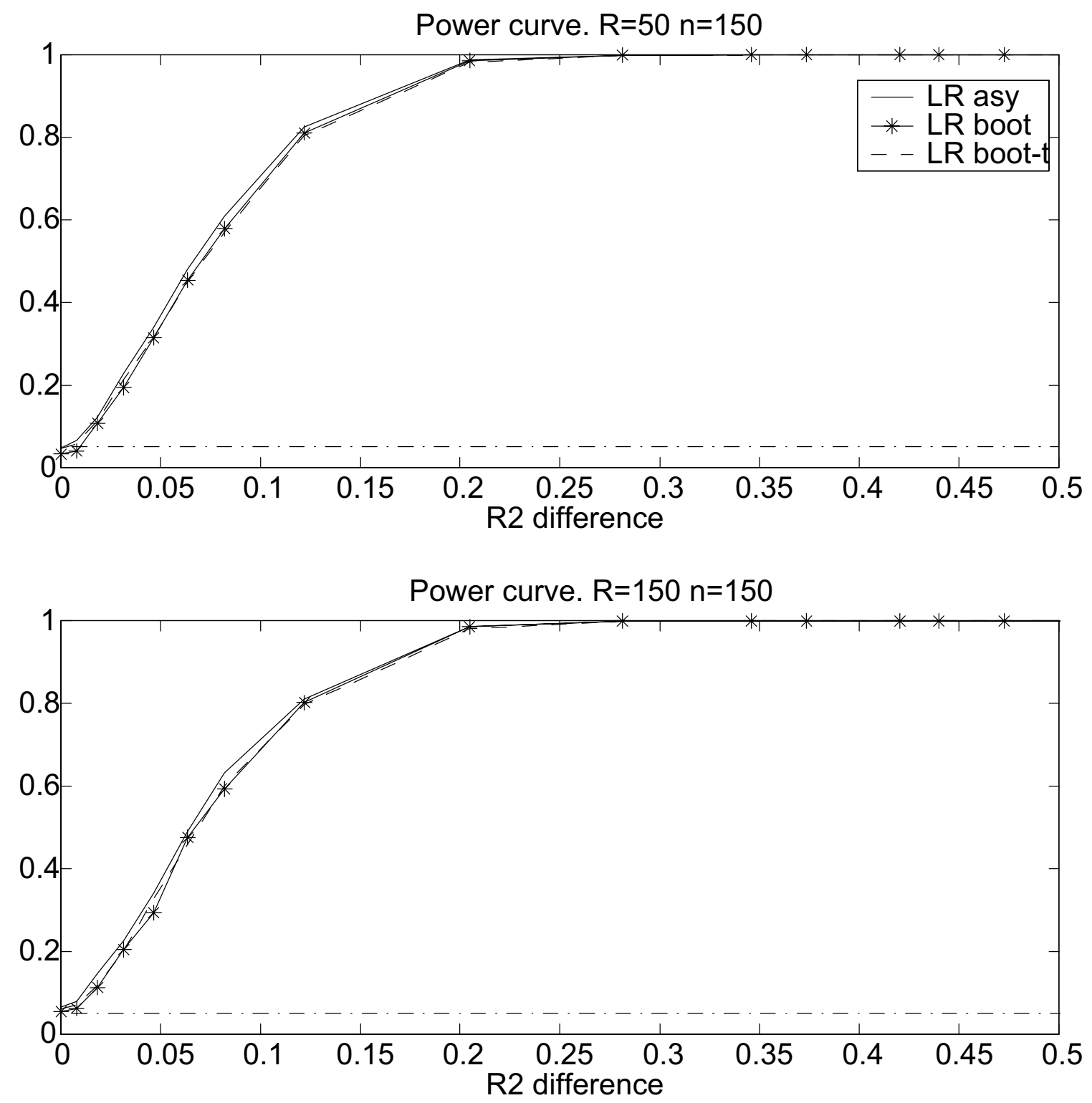

Figure 4: Power curves of LR asymptotic test, LR bootstrap test and LR bootstrap-t test in the Monte Carlo experiment discussed in Section 6. Each curve represents the rejection frequencies over 1000 Monte Carlo replications of the null hypothesis $H_{0}: E\left[\log g\left(Y_{t+1} \mid \Omega_{t} ; \gamma^{*}\right)-\log f\left(Y_{t+1} \mid \Omega_{t} ; \theta^{*}\right)\right]=0$, where the density forecasts $f, g$ and the DGP are defined in (15). The horizontal axis shows the difference in $R^{2}$ from the regressions defining the two density forecasts $f$ and $g$. Both figures consider an out-of-sample size of $n=150$. The upper panel is for in-sample size $R=50$ and the lower panel for $R=150$. 

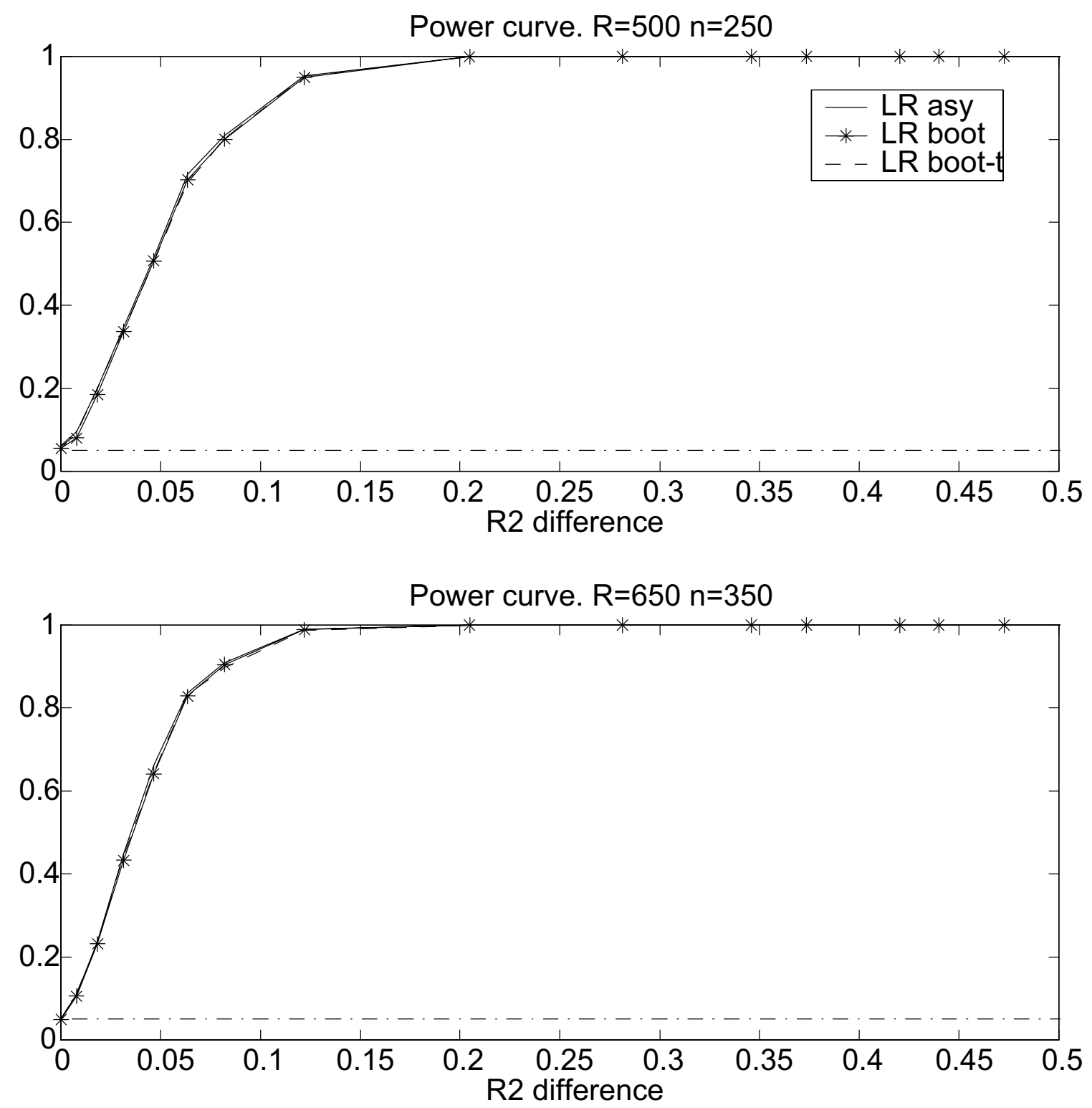

Figure 5: Power curves of LR asymptotic test, LR bootstrap test and LR bootstrap-t test in the Monte Carlo experiment discussed in Section 6. Each curve represents the rejection frequencies over 1000 Monte Carlo replications of the null hypothesis $H_{0}: E\left[\log g\left(Y_{t+1} \mid \Omega_{t} ; \gamma^{*}\right)-\log f\left(Y_{t+1} \mid \Omega_{t} ; \theta^{*}\right)\right]=0$, where the density forecasts $f, g$ and the DGP are defined in (15). The horizontal axis shows the difference in $R^{2}$ from the regressions defining the two density forecasts $f$ and $g$. The upper panel is for the pair of in-sample and out-of-sample sizes $R=500$ and $n=250$ and the lower panel is for $R=650$ and $n=350$. 


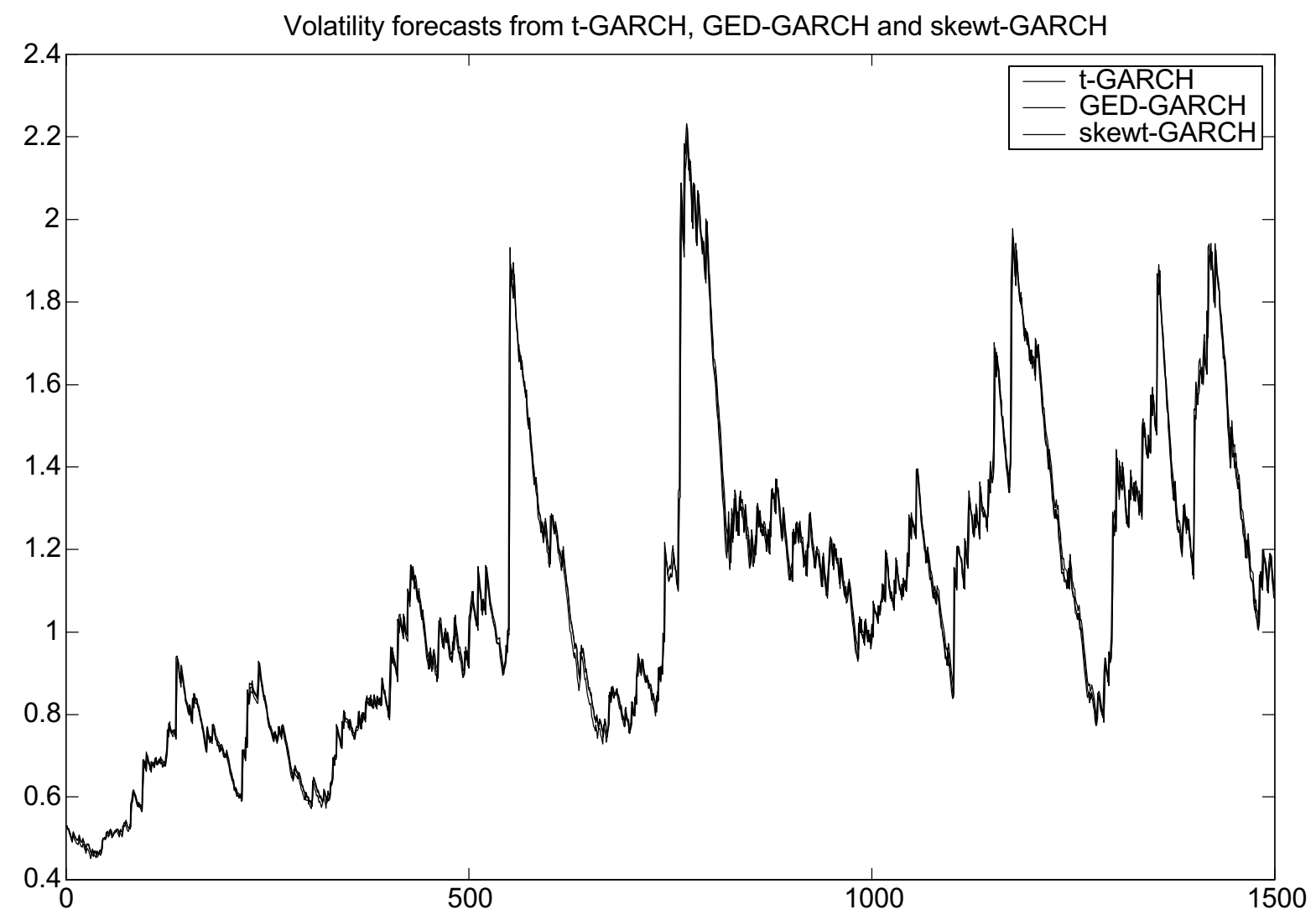

Figure 6: One-step-ahead forecasts of the daily volatility of the S\&P500 returns implied by recursively estimated $t-\operatorname{GARCH}(1,1), G E D-\operatorname{GARCH}(1,1)$ and skewt-GARCH$(1,1)$ models. 


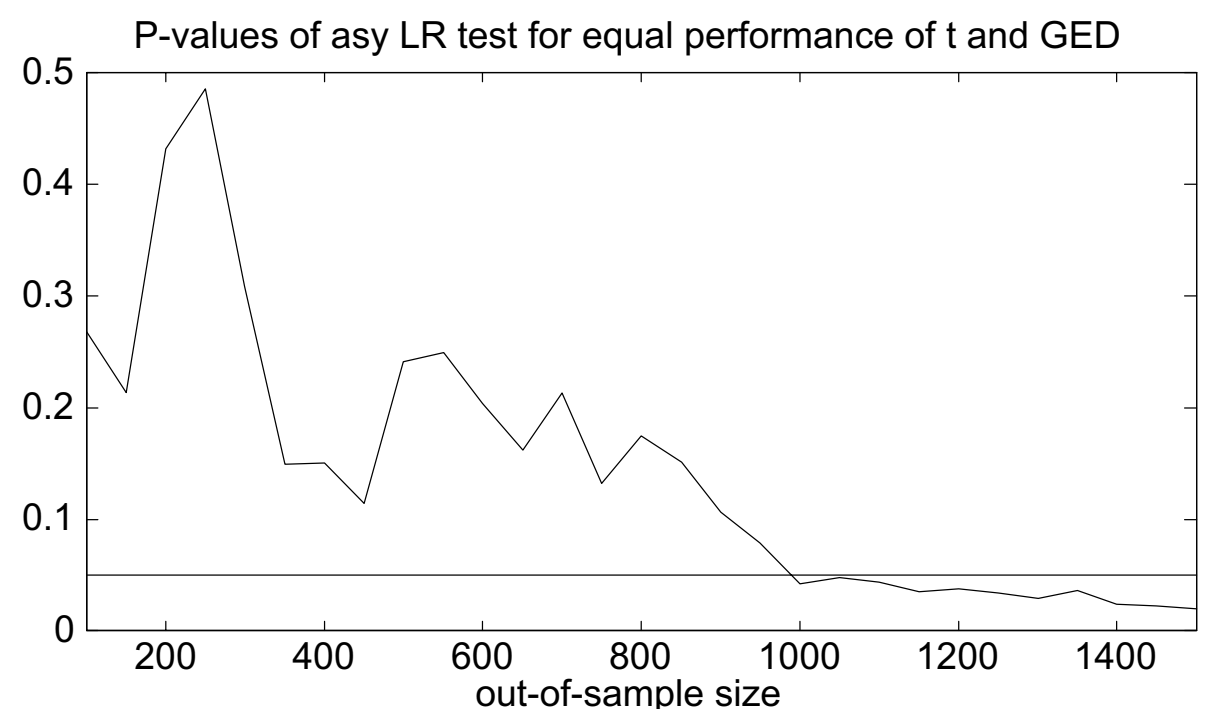

Figure 7: The figure shows p-values for the asymptotic LR test of the null hypothesis of equal performance of $t-\mathrm{GARCH}$ and $G E D-\mathrm{GARCH}$ density forecasts. The horizontal axis indicates increasing out-of-sample size.

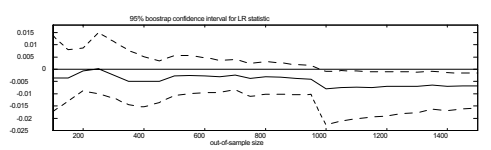

Figure 8: The solid line in the figure indicates the value of the test statistic $L R_{n}$, representing the out-of-sample mean of the logarithmic score differences for a $G E D-\mathrm{GARCH}$ density forecast and a $t-\mathrm{GARCH}$ forecast. A negative value indicates that $t-\mathrm{GARCH}$ outperforms $G E D-\mathrm{GARCH}$. The horizontal axis represents increasing out-of-sample size. The dashed line is the $95 \%$ bootstrap- $t$ confidence interval for $L R_{n}$. 

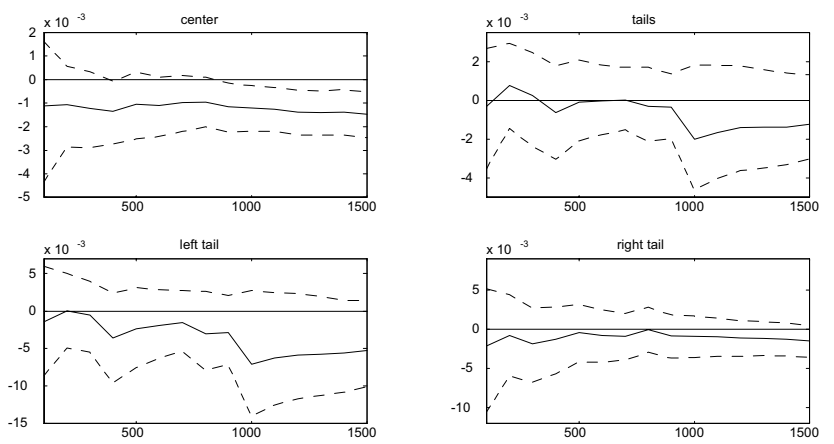

Figure 9: Weighted likelihood ratio tests of equal performance of $t-\mathrm{GARCH}$ and $G E D-\mathrm{GARCH}$ density forecasts. The figure shows $95 \%$ bootstrap confidence intervals for the weighted likelihood ratio statistics $W L R_{n}$ for increasing out-of-sample size. A negative value of the statistic indicates that $t-\mathrm{GARCH}$ outperforms $G E D-\mathrm{GARCH}$. The panels represent the four different weight functions shown in Figure 6; clockwise, the weight function used are $w_{1}, w_{2}, w_{3}$ and $w_{4}$. 


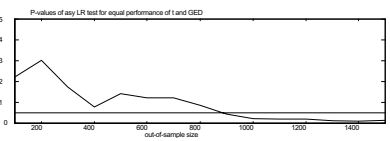

Figure 10: The figure shows p-values for the asymptotic LR test of the null hypothesis of equal performance of skewt-GARCH and GED-GARCH density forecasts. The horizontal axis indicates increasing out-of-sample size.

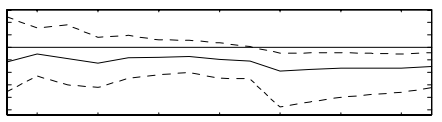

Figure 11: The solid line in the figure indicates the value of the test statistic $L R_{n}$, representing the out-of-sample mean of the likelihood ratio between a $G E D-G A R C H$ density forecast and a skewt-GARCH forecast. A negative value indicates that skewt-GARCH outperforms GED-GARCH. The horizontal axis represents increasing out-of-sample size. The dashed line is the $95 \%$ bootstrap- $t$ confidence interval for $L R_{n}$. 

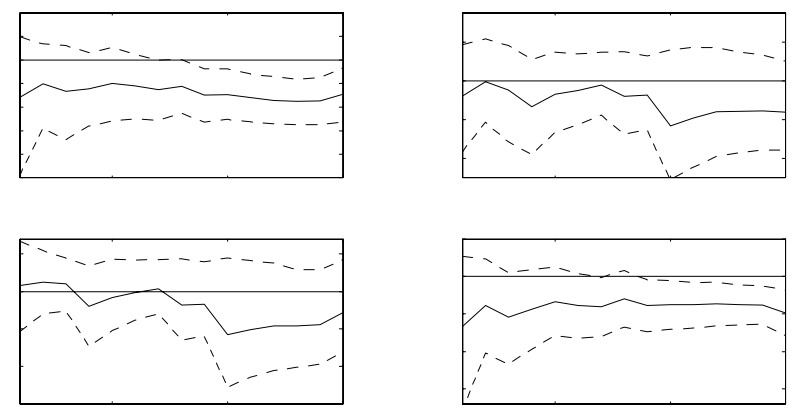

Figure 12: Weighted likelihood ratio tests of equal performance of skewt-GARCH and GED-GARCH density forecasts. The figure shows $95 \%$ bootstrap confidence intervals for the weighted likelihood ratio statistics $W L R_{n}$ for increasing out-of-sample size. A negative value of the statistic indicates that skewt-GARCH outperforms GED-GARCH. The panels represent the four different weight functions shown in Figure 6; clockwise, the weight function used are $w_{1}, w_{2}, w_{3}$ and $w_{4}$. 